Draft version August 29, 2018

Preprint typeset using LATEX style emulateapj v. 8/13/10

\title{
ANATOMY OF HELICAL EXTRAGALACTIC JETS: THE CASE OF S5 0836+710
}

\author{
M. PERUCHO ${ }^{1,3}$ \\ ${ }^{1}$ Departament d'Astronomia i Astrofísica, Universitat de València, 46100, Burjassot (València), Spain
}

\author{
Y.Y. KOVALEV ${ }^{1,2,3}$ \\ ${ }^{2}$ Astro Space Center of Lebedev Physical Institute, 117997 Moscow, Russia \\ A.P. LOBANOV ${ }^{3}$ \\ ${ }^{3}$ Max-Planck-Institut für Radioastronomie, Auf dem Hügel 69, 53121 Bonn, Germany \\ P.E. HARDEE ${ }^{4}$ \\ ${ }^{4}$ Department of Physics \& Astronomy, The University of Alabama, Tuscaloosa, AL 35487, USA \\ I. $\operatorname{AgUdO}^{5,6}$ \\ ${ }^{5}$ Instituto de Astrofísica de Andalucía, Apartado 3004, 18080, Granada, Spain and \\ ${ }^{6}$ Institute for Astrophysical Research, Boston University, 725 Commonwealth Avenue, Boston, MA 02215, USA \\ Draft version August 29, 2018
}

\begin{abstract}
Helical structures are common in extragalactic jets. They are usually attributed in the literature to periodical phenomena in the source (e.g., precession). In this work, we use VLBI data of the radio-jet in the quasar S5 $0836+710$ and hypothesize that the ridge-line of helical jets like this corresponds to a pressure maximum in the jet and assume that the helically twisted pressure maximum is the result of a helical wave pattern. For our study, we use observations of the jet in S5 $0836+710$ at different frequencies and epochs. The results show that the structures observed are physical and not generated artificially by the observing arrays. Our hypothesis that the observed intensity ridge-line can correspond to a helically twisted pressure maximum is confirmed by our observational tests. This interpretation allows us to explain jet misalignment between parsec and kiloparsec scales when the viewing angle is small, and also brings us to the conclusion that high-frequency observations may show only a small region of the jet flow concentrated around the maximum pressure ridge-line observed at low frequencies. Our work provides a potential explanation for the apparent transversal superluminal speeds observed in several extragalactic jets by means of transversal shift of an apparent core position with time.
\end{abstract}

Subject headings: galaxies: jets - hydrodynamics - instabilities - quasars: individual: S5 0836+710

\section{INTRODUCTION}

Jets in Active Galactic Nuclei (AGN) involve some of the most energetic processes in the Universe. The way they propagate and interact with the ambient medium and, in particular, their stability properties have been thoroughly studied (see Hardee 2006, 2011; Perucho 2011, for reviews). These jets are subject to the growth of different instabilities and many of the structures observed like knots, bendings, helices, have been interpreted as a result of this physical process. Although there could be other ways to produce these structures, such as interactions with clumps of dense gas or precession of the central engine, these in turn can also give rise to the growth of the instabilities via coupling to any of the unstable modes (see, e.g. Perucho et al. 2005; Mizuno et al. 2007; McKinnev \& Blandford 2009; Mizuno et al. 2009; Mignone et al. 2010; Perucho et al. 2010; Mizuno et al. 2011, for numerical studies of coupling to Kelvin-Helmholtz and current-driven instabilities in relativistic flows). Our interest in understanding

mailto: manel.perucho@uv.es how this process works in jets lies in the possibility of obtaining the physical parameters of the flow (velocity, gas density and sound speed), via modeling the observed structures (e.g., Lobanov \& Zensus 2001; Hardee et al. 2005; Hardee \& Eilek 2011). The Very Long Baseline Interferometry (VLBI) technique has provided highresolution observations that produce highly detailed images of the structure of AGN jets, and that even resolves them transversally (Lobanov \& Zensus 2001).

Radio images of many extragalactic jets from AGN present helical patterns on different spatial scales. There are a number of possible processes that can cause these structures, such as periodicity in the direction of ejection of the flow due to precession. Precession can be produced by the gravitational effect of the accretion disk on the compact object in the center of the AGN or in a binary-black-hole object (see, e.g., Lister et al. 2003; Stirling et al. 2003; Lobanov \& Roland 2005; Bach et al. 2006; Savolainen et al. 2006). If this periodical motion or any other initial perturbation of the relativistic jet couples to an unstable mode of a magneto-hydrodynamical instability like Kelvin-Helmholtz (KH, Perucho et al. 
2005; Mizuno et al. 2007; Perucho et al.|2010) or current driven (CD Mizuno et al. 2009; McKinney \& Blandford 2009; Mignone et al. 2010; Mizuno et al. 2011) instabilities, the perturbation may grow in amplitude as it is advected downstream with the jet flow. Thus, the growing helices can give us indirect information about their triggering process or eventually, about the physical parameters of the flow.

Hardee (2003) compared the intrinsic and observed nature of helical structures via pseudo-synchrotron images of artificially built helical jets from linear $\mathrm{KH}$ theory, following Hardee (2000). The results of this work were applied to the jet in $3 \mathrm{C} 120$ and allowed estimates to be made of some of the physical parameters of the jet. This work was extended in Hardee et al. (2005), where the authors constrained the jet parameters more tightly and reproduced the basic observed structure of 3C 120 by modeling the helical structures as $\mathrm{KH}$ modes. This type of analysis has also been applied to the jet in M 87 by Hardee \& Eilek (2011) who were able to constrain physical parameters in the jet and surrounding cocoon. Recently, Caproni et al. (2009) have proposed a method (cross-entropy optimizer) to fit helical patterns produced by precession in jets. The parameters obtained by this method are the jet velocity, the jet viewing angle, and the jet opening angle. The authors generated artificial data-sets, which were successfully fitted by their method.

Another problem related to observations of VLBI-jets is the measurement of superluminal transversal motion of components. Gong (2008) has proposed non-ballistic motion of physical components and misidentification as the origin of those measurements, although the latter is not a valid explanation for the case of densely time sampled jets (e.g.,the case of NRAO 150 and OJ287, Agudo et al. 2007, 2010, 2011). In this context, it is interesting to note that components fitted in radio-jets do not necessarily represent physical entities, but just a way to model the continuous structure of those jets. Only in some cases it is possible to associate the components that have been fitted to physical structures, such as perturbations crossing the jet or standing features, both usually related to shocks in the literature.

In this paper, we present a deep analysis of multiwavelength and multi-epoch observations of the helical jet in S5 $0836+710(0836+710$ hereafter $)$. Our analysis is based on the assumption that the maximum intensity of the radio emission coincides with the location of the pressure maximum in the jet. This is a reasonable assumption if the velocity of the emitting region of the flow is fairly symmetric and there are no strong beaming asymmetries across the jet. The pseudo-synchrotron images produced in Hardee (2003), Hardee et al. (2005) and Hardee \& Eilek (2011) also indicate that the maximum intensity in helically perturbed jets comes from the location where the pressure is at its maximum. Here, we present different tests that confirm this point from the observational data. Our analysis does not put any constraint on the origin of the helices, but we hypothesize that the initial helical motion couples to either a $\mathrm{KH}$ or CD instability.

The luminous quasar S5 $0836+710$ at a redshift $z=$ 2.16 hosts a powerful radio jet extending up to kiloparsec scales (Hummel et al. 1992). At this redshift, 1 mas $\simeq 8.4 \mathrm{pc}$ (see MOJAVE database and Wright
2006). VLBI monitoring of the source (Otterbein et al. 1998) has yielded estimates of the bulk Lorentz factor $\gamma_{\mathrm{j}}=12$ and the viewing angle $\alpha_{\mathrm{j}}=3^{\circ}$ of the flow. The presence of an instability developing in the jet is suggested by the kink structures observed on milliarcsecond scales with ground VLBI (Krichbaum et al. 1990). Lobanov et al. (1998) observed the source at $5 \mathrm{GHz}$ with VSOP2 and also reported oscillations of the ridge-line. Identifying these structures with $\mathrm{KH}$ modes, they were able to derive an estimate of the basic parameters of the flow. High dynamic range VSOP and VLBA (Very Long Baseline Array of National Radio Astronomy Observatory, USA) observations of $0836+710$ at $1.6 \mathrm{GHz}$ indicated the presence of an oscillation at a wavelength as long as 100 mas (Lobanov et al. 2006), which cannot be readily reconciled with the jet parameters given in Lobanov et al. (1998). Perucho \& Lobanov (2007) have shown that the presence of a shear layer allows fitting all the observed oscillation wavelengths within a single set of parameters, assuming that they are produced by $\mathrm{KH}$ instability growing in a cylindrical outflow. In this picture, the longest mode corresponds to a surface mode growing in the outer layers, whereas the shorter wavelengths are identified with body modes developing in the inner radii of the jet. This result is reviewed here, in the light of additional information.

The paper is structured as follows. In Section 2 we present the results from observations that will be used in later sections for our study. In Section 3 we describe the implications deduced from this set of multi-frequency and multi-epoch observations. In Section 4 we present a model to fit the ridge-line structures in relativistic jets. In Section 5 we discuss some properties of the jet inferred from the observations. Section 6 is devoted to the nature of the observed superluminal transversal motions in parsec-scale AGN jets in the context of our work. Finally, we present our summary in Section 7.

\section{MULTI-EPOCH AND MULTI-FREQUENCY OBSERVATIONS OF THE JET IN $0836+710$}

Observations of the jet at different frequencies and epochs are critical to our study. The different frequencies provide information on the structures arising at different spatial scales within the jet (Perucho \& Lobanov 2007). The different epochs provide a means to determine structure motions. Table 1 shows the frequencies and epochs that have been used in this work: VLBA and VSOP at 1.6 and $5 \mathrm{GHz}$ (Lobanov et al. 2006) at three and two different epochs, respectively, two epochs at $1.6 \mathrm{GHz}$ from $\mathrm{EVN}^{3}$, one epoch of simultaneous global VLBI (including VLBA) observations at 2 and $8 \mathrm{GHz}$ (01/1997, Pushkarev \& Kovalev, submitted), two epochs from VLBA at $8 \mathrm{GHz}$, three epochs from VLBA at 22 and $43 \mathrm{GHz}$, and 13 epochs, between 1998 and 2009, from the

\footnotetext{
$1 \quad$ https://www.physics.purdue.edu/astro/mojave/, which uses $\triangle C D M$ Cosmology from WMAP 5 year results, and Ned Wrights Cosmology Calculator http://www.astro.ucla.edu/ wright/CosmoCalc.html

VLBI Space Observatory Program, a Japanese-led space VLBI mission operated in 1997-2007 by the Institute of Space and Astronautical Science, Sagamihara, Japan http://www.vsop.isas.jaxa.jp/top.html

3 The European VLBI Network is a joint facility of European, Chinese, South African and other radio astronomy institutes funded by their national research councils.
} 
$2 \mathrm{~cm}$ VLBA/MOJAVE database at $15 \mathrm{GHz}$. Figures 1 to 3 show several of these maps.

The top two panels of Figure 1 show observations at 1.6 $\mathrm{GHz}$ separated by about 10.17 yr but using two different instruments. There is clear indication of a long wavelength oscillation and it can be seen that there are no significant changes at these large scales over this period. The bottom two panels show observations at $5 \mathrm{GHz}$ separated by about $6.83 \mathrm{yr}$, both with the VLBA. There is some evidence for a long wavelength oscillation in both images but there are significant changes to the structure with a suggestion of a shorter wavelength oscillation in the 2003 epoch at larger core distance (right bottom panel).

The top three panels of Figure 2 show observations at $8 \mathrm{GHz}, 19$ and 16 months apart, spanning about $3 \mathrm{yr}$. The first image was obtained using Global-VLBI but the subsequent two images were obtained with the VLBA. These panels show only negligible difference, so there is little change in the jet structure over this $3 \mathrm{yr}$ period. The bottom three panels show observations at $15 \mathrm{GHz}$, separated by 40 and 43 months and spanning about $7 \mathrm{yr}$. All three show evidence for some long wavelength oscillation, longer than the observed jet, that does not change significantly over this period. The only significant difference is that the first image clearly shows a shorter wavelength oscillation far from the core, whereas this shorter wavelength oscillation is weak at later times.

The top two panels of Figure 3 show observations at $22 \mathrm{GHz}$ with $1.5 \mathrm{yr}$ separation. Both show a straight jet along the first miliarcsecond, with some curvature appearing in the second image at larger distances from the core. The bottom two panels show observations at $43 \mathrm{GHz}$ with the same $1.5 \mathrm{yr}$ separation and show a curved jet in the first epoch and a straighter structure in the second epoch. Note that the curved structure in the first epoch at $43 \mathrm{GHz}$ could propagate out to larger scale with time and appear as the curved jet at larger scale in the $22 \mathrm{GHz}$ second epoch. This would require a motion of about 0.2 mas in $1.5 \mathrm{yr}(0.13 \mathrm{mas} / \mathrm{yr} \simeq 3.5 c$ apparent velocity). If the change in curvature were related to one quarter of a wave period, it would imply an observed periodicity of $\sim 6 \mathrm{yr}$.

The ridge-line of the jet was obtained by determining the center of the jet emission (fitted by a Gaussian) at a given radial distance from the core. This calculation was performed at the different epochs and frequencies. It was done radially outwards to obtain a complete picture of the ridge-line of the jet. The computed ridge-lines are indicated as red diamonds on Figures 1 1 3.

We have tested possible deviations in the computed ridge-line by obtaining it using two other approaches: from the location of the maximum emission and from the geometrical center of the emission profile in a transversal slice above a certain image rms cutoff level. As an example, Figure 4 shows the ridge-lines found by the three different methods for the $1.6 \mathrm{GHz}$ image of the jet in 1997 (also shown in Fig. 1). The lines are overlaid for comparison and we find that there are no significant differences between the geometrical center of the emission profile, position of maximum emission and the center of jet emission as found by a Gaussian fit (as that shown in Fig. 11). This coincidence will be reviewed below in the light of higher resolution observations at $15 \mathrm{GHz}$.
In these comparisons, errors in the displacements are assumed to be one fifth of the FWHM of the resolving beam in the slice direction (this assumption is also used throughout the paper). We note that, in our specific case, this is a conservative assumption, as the positional errors are expected to be $\sim 2 \mathrm{FWHM} / \mathrm{SNR}$ (where SNR described the peak-to-rms signal to noise in the slice; $c f$., Condon 1997). SNR $\geq 10$ is obtained in all slices covering the entire relevant section of the jet, a conservative error estimate is justified to be used for the purpose of comparing the ridge lines obtained by the three different methods. With this approach, we find that all three methods are consistent with each other, hence implying that the resulting individual ridge lines are different by less than $20 \%$ of the FWHM. A more detailed measure of positional errors of the ridge-line, taking into account the effect of the SNR and the transverse width of the jet, will be employed in future work (Perucho et al., in preparation) in order to provide accurate comparisons with predictions from theoretical models.

\section{OBSERVATIONAL EVIDENCE}

In this section we discuss the ridge-line positions at different frequencies and epochs. In general, the apparent position of the opaque core depends on observing frequency and can shift with frequency on the sky (Lobanov 1998). However, we align images by apparent position of the core and do not take this effect into account while comparing the ridge-lines. This is justified by the small expected shifts ( $\leq 1$ mas) as compared to the length of the structures that are compared (typically tens of mas). See for typical amount of the shift the statistical studies by Kovalev et al. (2008) and Sokolovsky et al. (2011).

\subsection{On the nature of ridge-lines}

Figures [5:7 display a combination of the radio emission contours for different frequencies along with their ridge-lines for observations performed in 1997, 1998 and 2003. Figure 5 shows that the ridge-lines indicated at successively higher frequencies appear as oscillations on top of the ridge-lines obtained at the lower frequencies: the green $5 \mathrm{GHz}$ and red $8 \mathrm{GHz}$ ridge-lines wrap around the blue $2 \mathrm{GHz}$ ridge-line. Differences between the 1.6 and $2 \mathrm{GHz}$ ridge-lines are within the errors.

Figure 6 shows the same kind of behavior at higher frequencies in 1998, with the longest ridge-line belonging to the $8 \mathrm{GHz}$ image and the ridge-lines at 15,22 and $43 \mathrm{GHz}$ appearing as oscillations on top of the $8 \mathrm{GHz}$ ridge-line.

Finally, Figure 7 shows the broadest range of frequencies, from 1.6 to $43 \mathrm{GHz}$, with the bottom panels showing an enlarged image of the inner region. Again, the ridgelines of the high-frequency images tend to lie on top of the lower frequency ridge-lines, except for the $15 \mathrm{GHz}$ image ridge-line that deviates significantly from the $1.6 \mathrm{GHz}$ ridge-line far from the core. However, at these distances, the signal-to-noise ratio is low in the $15 \mathrm{GHz}$ image, which should translate into larger errors, and the difference is always within the error of the $1.6 \mathrm{GHz}$ ridge-line $(\simeq 1$ mas $)$.

In summary we find that the ridge-lines show remarkable coincidence independent of the epoch and the array. Significant differences would be expected if oscillation of 
TABLE 1

ObSERVATION Epochs AND Frequencies

\begin{tabular}{|c|c|c|c|c|c|c|c|}
\hline YEAR & $1.6 \mathrm{GHz}$ & $2 \mathrm{GHz}$ & $5 \mathrm{GHz}$ & $8 \mathrm{GHz}$ & $15 \mathrm{GHz}$ & $22 \mathrm{GHz}$ & $43 \mathrm{GHz}$ \\
\hline 1997 & VLBA & G-VLBI & VLBA & G-VLBI & . & . & . \\
\hline 1998 & VLBA & $\ldots$ & $\ldots$ & VLBA & VLBA & VLBA & VLBA \\
\hline 1999 & ... & $\ldots$ & $\ldots$ & VLBA & VLBA & VLBA & VLBA \\
\hline 2000 & $\ldots$ & $\ldots$ & $\ldots$ & $\ldots$ & VLBA & $\ldots$ & $\ldots$ \\
\hline 2001 & $\ldots$ & $\ldots$ & $\ldots$ & $\ldots$ & VLBA & $\ldots$ & $\ldots$ \\
\hline 2002 & $\ldots$ & $\ldots$ & $\ldots$ & $\ldots$ & VLBA & $\ldots$ & $\ldots$ \\
\hline 2003 & VLBA & $\ldots$ & VLBA & $\ldots$ & VLBA & VLBA & VLBA \\
\hline 2004 & $\ldots$ & $\ldots$ & $\ldots$ & $\ldots$ & VLBA & $\ldots$ & $\ldots$ \\
\hline 2005 & $\ldots$ & $\ldots$ & $\ldots$ & $\ldots$ & VLBA & $\ldots$ & $\ldots$ \\
\hline 2006 & $\ldots$ & $\ldots$ & $\ldots$ & $\ldots$ & VLBA & $\ldots$ & $\ldots$ \\
\hline 2007 & EVN & $\ldots$ & $\ldots$ & $\ldots$ & VLBA & $\ldots$ & $\ldots$ \\
\hline 2008 & EVN & $\ldots$ & $\ldots$ & $\ldots$ & VLBA & $\ldots$ & $\ldots$ \\
\hline 2009 & $\ldots$ & $\ldots$ & $\ldots$ & $\ldots$ & VLBA & $\ldots$ & $\ldots$ \\
\hline
\end{tabular}

the ridge-line were array or epoch-dependent. This result confirms the real nature of the structures observed in extragalactic jets by means of VLBI observations.

If the jet is transversally resolved, the maximum in the emission, or the position of the ridge-line can be due to two main factors: 1) differential Doppler boosting caused by velocity gradients in the jet, or 2) an increase in the local pressure and number of emitting particles. If the position of this maximum is due to differential Doppler boosting, changes in the flow direction would have an influence on the local emission from the different regions. This should result, depending on the internal velocity gradient, in irregular patterns, contrary to the results obtained. In addition, relatively large changes in speed and/or flow direction are required in order to get significant differential Doppler boosting within the jet. Thus, the most reasonable hypothesis is that the ridge-line corresponds to a pressure maximum. In the following sections we present evidence that supports this interpretation.

\subsection{Ridge-lines and transversal structure}

A straight axis can be defined for the jets, by a line starting at the core and defined by the symmetry axis of the the longest-observed wavelength oscillation (assumed to result from helical motion). Note that this line, which will be referred to as the jet axis or simply the axis, is not the same as the center or the maximum of the jet emission at each radial distance, used above to determine the position of the ridge-line. Within our interpretation, the axis describes a mean or reference direction of the jet the direction of flow propagation in absence of instabilities. This axis needs to be defined in order to be able to estimate the amplitudes and wavelength of individual instability modes, as done in Lobanov \& Zensus (2001). The mean overall jet direction $\chi$, as defined by the jet axis, is modified by a helical instability (see Hardee 2000, for a discussion of the effect of different $\mathrm{KH}$ modes on jet structure and next sections). Variations of the ridge lines observed on smaller scales are likely to be caused by shorter unstable wavelengths. In order to estimate accurately wavelengths and amplitudes of these oscillations, the effect of the longest wavelength has to be taken into account, either by modeling it with an oscillatory term or by assuming a mean local jet direction at each relevant spatial scale. As the small-scale ridge line variations occur on scales substantially shorter than the longest observed wavelength, we assume local mean jet directions $\chi_{\nu}$ for observations at different frequencies (relying on the fact that the spatial scales investigated depend largely on the observing frequency). If the jet axis is computed locally, for the different frequencies, the comparison shows that the absolute value of the jet position angle $\left(\chi_{\nu}\right)$ increases with the frequency, where this angle is measured from the Declination axis, with $0^{\circ}$ corresponding to the positive values.

The angle $\chi_{\nu}$ does not change with time at each frequency. Figures 8 and 9 show this effect, with the position angle of the jet ranging from $198^{\circ}$ at $1.6 \mathrm{GHz}$ to $214^{\circ}$ at $22 \mathrm{GH}$, indicated by the dashed blue lines. The values of the different position angles at the different frequencies are listed in Table 2.

Recall that there is a clear superposition of high frequency ridge-lines on top of the low frequency ridge-lines (Figs. [5]7). This can be interpreted as the radio emitting regions at high-frequencies being concentrated in a relatively small region around the lower frequency ridgeline with the small scale oscillations seen at higher frequency developing on top of the long scale ones seen at lower frequency. This interpretation provides a natural explanation of parsec-to kiloparsec scale jet misalignment for helically twisted jets pointing close to the line of sight. However, this raises a question as to whether the lowest-frequency emission observed is embedded in an even broader region, with lower or no emission. If this was the case, we would not be observing the whole crosssection of the jet at high-frequencies, but only a small region around the highest-emitting locations. Thus, the apparent helix would be tracing a high-emission region inside the jet, but not the whole jet.

To test the possibility of a frequency dependent transversal structure in the emitting region, we computed the jet observed opening angles and widths for all the available frequencies at different positions along the jet axis following Pushkarev et al. (2009). Note that, as stated above, the direction of the observed jet changes with the frequency and this should be taken into account to obtain these parameters at each frequency. Thus, the appropriate position angle of the frequency-dependent axis, $\chi_{\nu}$, was used for each frequency. The results of this analysis are summarized in Table 2 , where average values

\footnotetext{
${ }^{4}$ It is impossible to determine the jet position angle at $43 \mathrm{GHz}$ due to its short length.
} 

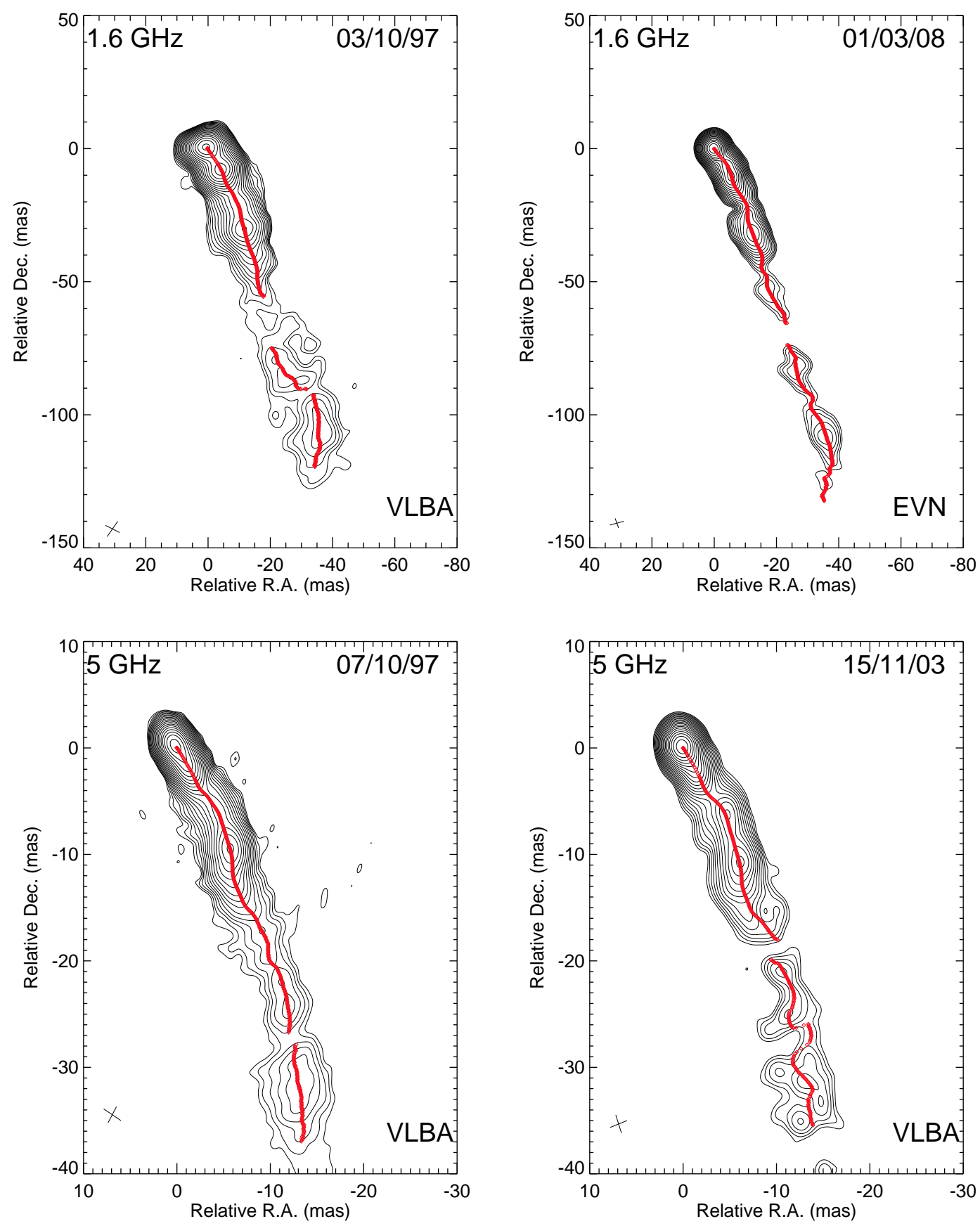

FIG. 1.- Radio map of the jet in $0836+710$ at $1.6 \mathrm{GHz}$ (top) in 1997 and 2008 and $5 \mathrm{GHz}$ (bottom) in 1997 and 2003 . Isocontours range from $2 \mathrm{mJy}$ to $1.45 \mathrm{Jy}$ in the $1.6 \mathrm{GHz}$ images and from $1.5 \mathrm{mJy}$ to $1.08 \mathrm{Jy}$ in the $5 \mathrm{GHz}$ ones. The red diamonds indicate the position of the ridge-line.

for each frequency are given. Not all epochs were used, as some were too noisy for this study. The errors come from the dispersion of the values for all epochs. We find that the opening angles are very similar at all frequencies, with an average value of $13.8^{\circ} \pm 1.9$. At the highest frequencies, 22 and $43 \mathrm{GHz}$, we have only considered one epoch (2003) due to problems with the other two epochs (1998, 1999). The resulting values are subject to larger errors as the opening angles are obtained with fewer cuts. If we disregard these high frequency results, the average opening angle is $12.1^{\circ} \pm 0.8$, which at a $3^{\circ}$ viewing angle implies an intrinsic opening angle of $0.63^{\circ} \pm 0.04$. This value coincides with that obtained at $15 \mathrm{GHz}$, for which we have the largest sample and best quality data. The small or negligible differences between the opening angles at different frequencies tells us that either all transversal regions in the jet expand at the same rate or that, with the present resolution it is still difficult to tell whether there is a transversal frequency profile for this jet.

\subsection{Possible secondary peak and implications}

Figure [10] shows profiles of the jet brightness at distances from the core between 6.4, 7.5 and 8.2 mas for different frequencies and epochs. These profiles do not 

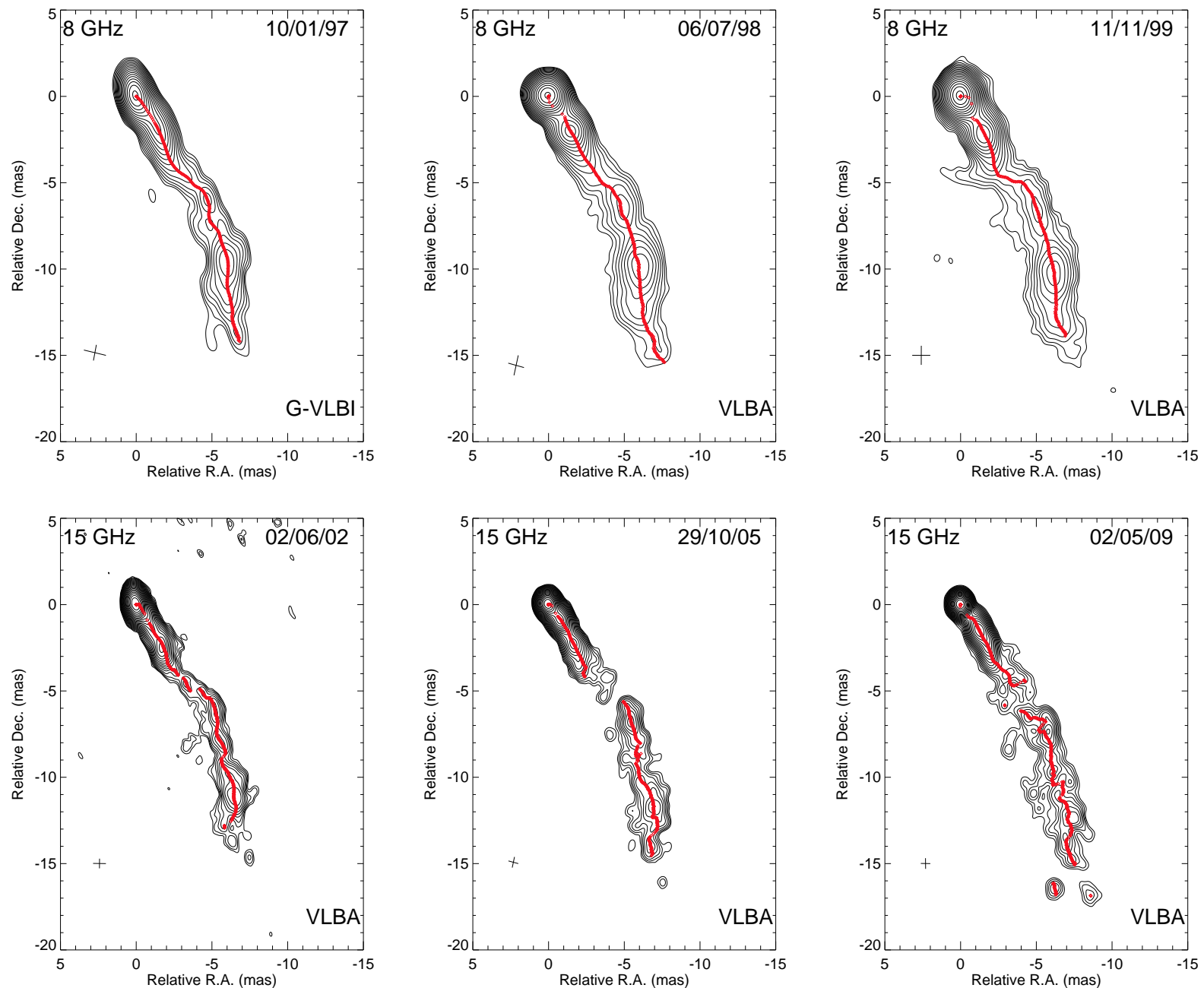

FIG. 2.- Same as 1 for $8 \mathrm{GHz}$ (top) in 1997, 1998 and 1999 and $15 \mathrm{GHz}$ (bottom) in 2002, 2005 and 2009. Isocontours range from 2.5 mJy to $1.8 \mathrm{Jy}$ in the $8 \mathrm{GHz}$ images and from $0.5 \mathrm{mJy}$ to $0.7 \mathrm{Jy}$ in the $15 \mathrm{GHz}$ ones.

TABLE 2

OBSERVED JET PROPERTIEs.

\begin{tabular}{lcccccc}
\hline \hline & $1.6 \mathrm{GHz}$ & $5 \mathrm{GHz}$ & $8 \mathrm{GHz}$ & $15 \mathrm{GHz}$ & $22 \mathrm{GHz}$ & $43 \mathrm{GHz}$ \\
\hline Average opening angle & $(12.3 \pm 1.2)^{\circ}$ & $(13.5 \pm 1.3)^{\circ}$ & $(12.1 \pm 0.8)^{\circ}$ & $(10.5 \pm 0.9)^{\circ}$ & $18.1^{\circ}$ & $16.6^{\circ}$ \\
Position angle $(\chi)$ & $198^{\circ}$ & $202^{\circ}$ & $206^{\circ}$ & $210^{\circ}$ & $214^{\circ}$ & - \\
Oscillation wavelengths (0-10 mas) & $10-80$ & 10 & 10 & 10 & - & - \\
Oscillation wavelengths (10-35 mas) & $20-80$ & 20 & $7-20$ & 20 & - & - \\
Oscillation wavelengths ( $>35$ mas) & $40-80$ & - & - & - & - & -
\end{tabular}

vary significantly with the position angle used, so we have taken $\chi_{8}$, i.e., the position angle of the jet at $8 \mathrm{GHz}$ as a characteristic value. The positions of these profiles are indicated in Fig. 17. In all the plots, the observed emission maximum is displaced (by $1-2$ mas) with respect to the reference axis, beyond the errors in the determination of the peak. We will see in Section 3.5 that the measured values of the relative shift between epochs deviate from zero due to possible evolution of the ridge-lines. In most cases, single Gaussians provide good fits to the profiles and the center of jet emission coincides with the computed ridge-line. However, Figure 10 shows that the transversal profiles at 8 and $15 \mathrm{GHz}$ contain a secondary peak at the relative offset of $-2--1$ mas, which is not seen at the other frequencies at the same position. In order to verify that the second peak is real, a stacked imag 5 from the MOJAVE database was used, and the same double peak structure in the profile was observed at this distance. In addition, if the image at $15 \mathrm{GHz}$ is restored with the resolution of the $5 \mathrm{GHz}$ data, the secondary peak disappears and the resulting profiles are very similar to the $5 \mathrm{GHz}$ ones (Fig. 10). Finally, the $5 \mathrm{GHz}$ image (shown in Fig. 1) is accurately reproduced by the map of the jet at $15 \mathrm{GHz}$ when convolved with

5 This image is a composition from all the epochs, which reinforces real features and smoothes noisy ones. 

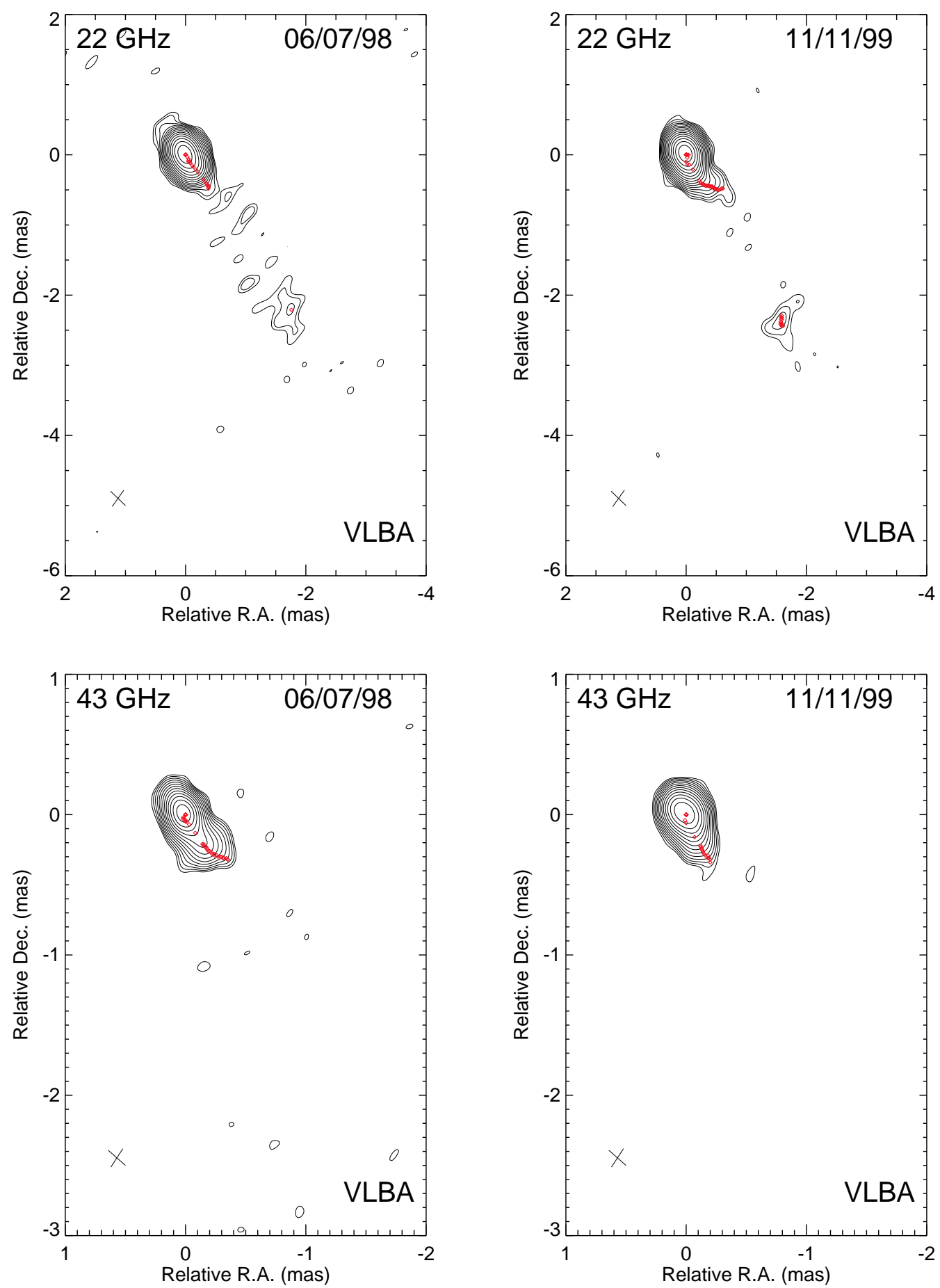

FIG. 3.- Same as 1 for $22 \mathrm{GHz}$ (top) and $43 \mathrm{GHz}$ (bottom) in 1998 and 1999. Isocontours range from $7 \mathrm{mJy}$ to $5 \mathrm{Jy}$ in the $22 \mathrm{GHz}$ images and from $8 \mathrm{mJy}$ to $5.8 \mathrm{Jy}$ in the $43 \mathrm{GHz}$ ones.

the $5 \mathrm{GHz}$ beam.

At $15 \mathrm{GHz}$, where the two peaks are observed, the stronger peak is slightly displaced from the center of the emitting region ( -3 to +4 mas, approximately), which is located around 2 mas to the right from the coordinate axis (jet axis, see Fig. 10). The secondary peak is less important than the one to the right. This structure could be explained by superposition of a smaller amplitude elliptical mode and a dominant helical mode, or by limb-brightening of the jet.

A growing instability in the linear regime implies a straight, expanding jet with the unstable pattern developing in its interior or on the surface/layer separating the jet from its surrounding medium (e.g., Hardee 2000; Perucho et al. 2006). Thus, within this frame, the center of the jet emission and the ridge-line associated to the maximum emission do not necessarily coincide, if the latter is produced by the pressure maxima. The local di- 

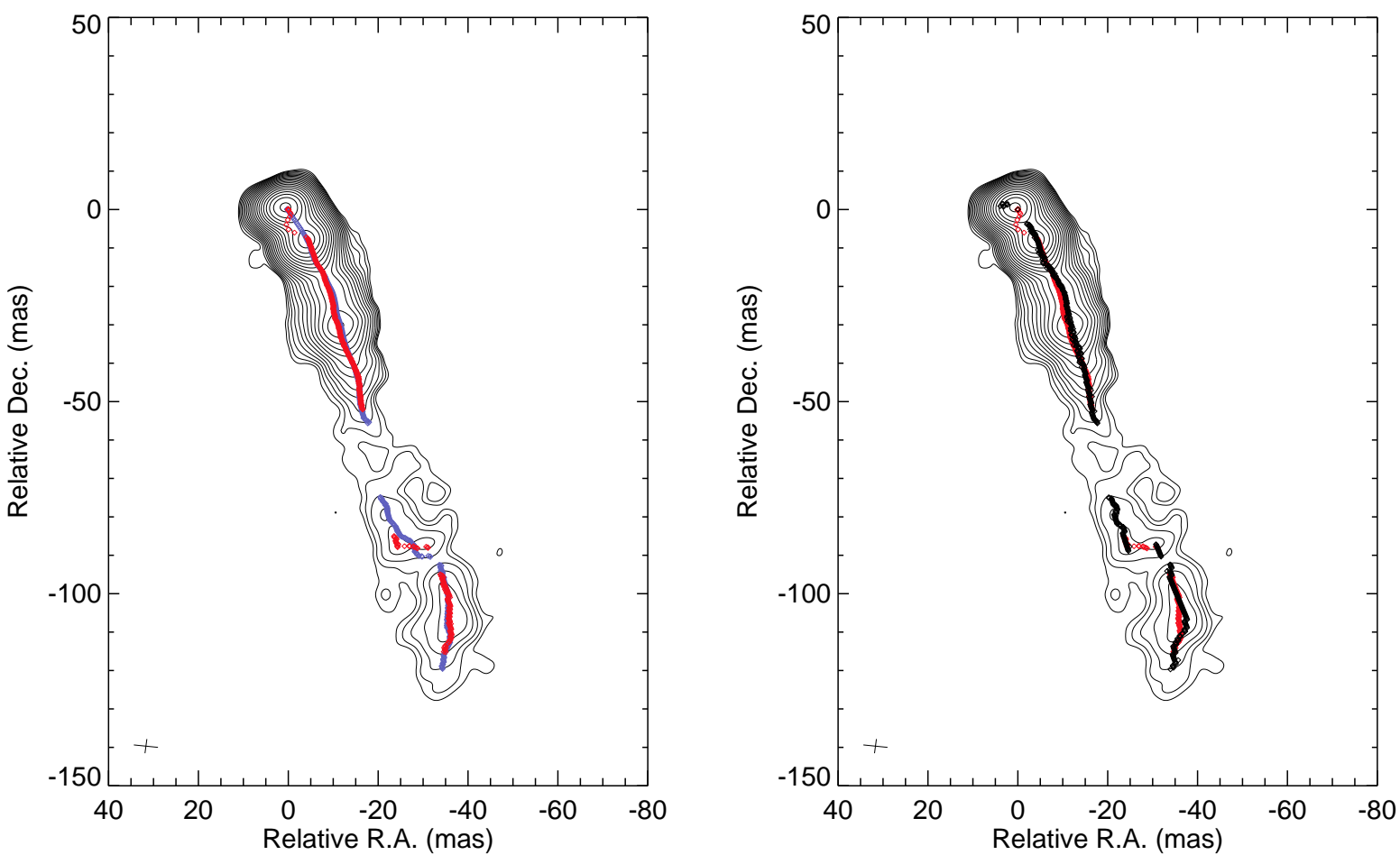

FIG. 4.- Radio map of the jet in $0836+710$ at $1.6 \mathrm{GHz}$ in 1997 . The left panel shows the comparison between the ridge-lines computed from a Gaussian fit (red diamonds) and the geometrical center of the jet emission profile (blue diamonds). The right panel shows the comparison with the position of the maximum emission (black diamonds).
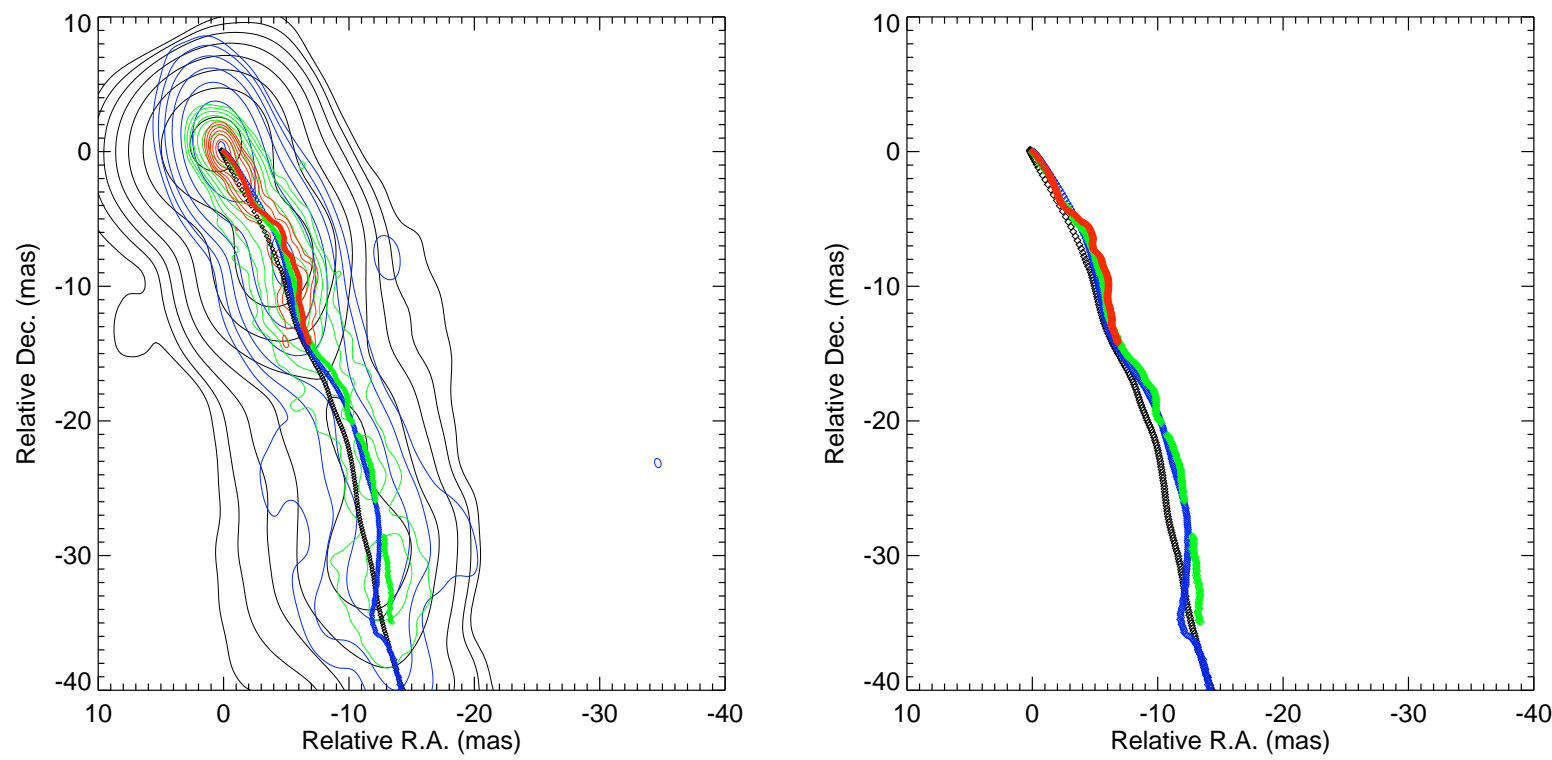

Fig. 5. - Radio maps at the 1.6 (black contours), 2 (blue contours), 5 (green contours) and $8 \mathrm{GHz}$ (red contours) of the jet in $0836+710$ in 1997. The ridge-lines at the different frequencies are indicated by diamonds in the same colour as the contours and are shown without contours on the right.

rection of the flow can change when the jet is affected by a helical mode and the maximum in pressure is displaced from the center of the flow, as shown in previous works (Hardee 2000; Perucho et al. 2006). The fact that the peak in emission at $15 \mathrm{GHz}$ (Figure 10) is shifted from the center of the emitting jet supports the idea that the peak in emission does not coincide with the center of the jet flow and is more related to pressure differences in its cross-section. However, only if the jet is resolved, this feature is fully captured. This is the reason why, at $1.6 \mathrm{GHz}$, where the jet is not well resolved, the different methods to determine the ridge-lines give similar results (see Section 2 and Figure 4). 

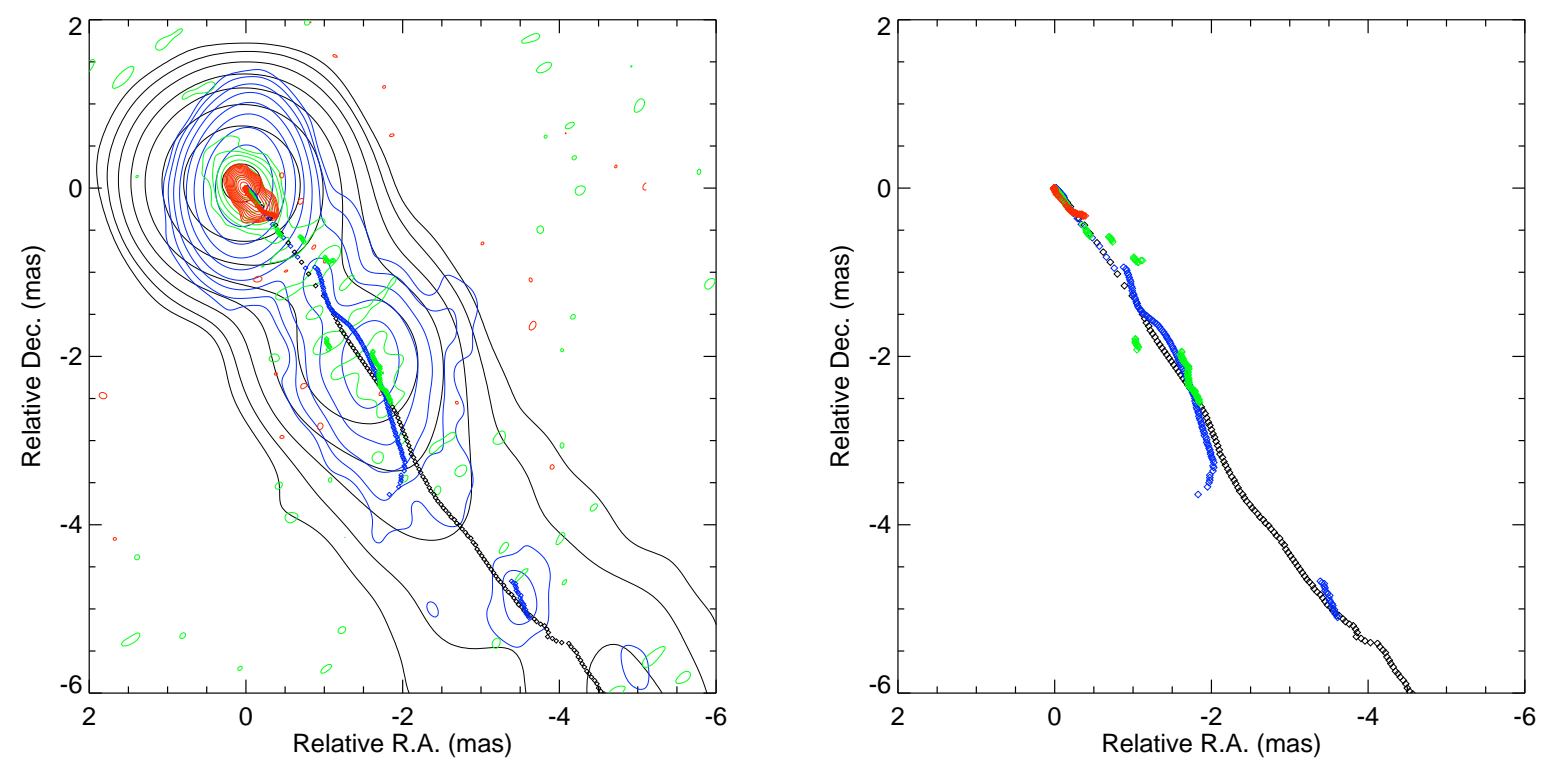

FIG. 6. - Radio maps at the 8 (black contours), 15 (blue contours), 22 (green contours) and $43 \mathrm{GHz}$ (red contours) of the jet in $0836+710$ in 1998; the ridge-lines at the different frequencies are indicated by diamonds in the same colour as the contours and are shown without contours on the right.

\subsection{Observed oscillation wavelengths}

Applying a rotation of the jet position angle to the coordinates of the ridge-line on the plane of the sky, the structure is brought to a horizontal axis (x-axis in cartesian coordinates) along the positive Declination axis, making the detection of patterns much easier:

$$
\begin{gathered}
x=x_{o b} \cos (2 \pi-\chi)-y_{o b} \sin (2 \pi-\chi) \\
y=x_{o b} \sin (2 \pi-\chi)+y_{o b} \cos (2 \pi-\chi),
\end{gathered}
$$

where $x_{o b}$ and $y_{o b}$ are the measured coordinates, $\chi$ is the position angle of the jet in the plane of the sky defined in Section 3 and $x$ and $y$ are the rotated coordinates. Note that once rotated, the axis $y_{o b}$ falls on the negative $y$ axis. Figure 11 shows the different coordinate systems used in this work. The coordinate system $x, y$ is only used for wavelength detection purposes. In the next sections we will only refer to $x_{o b}, y_{o b}$ (observed positions) and $x_{h}, y_{h}, z_{h}$ (intrinsic coordinate system).

Figure 12 shows the transverse location of the ridgelines relative to the jet axis $\left(\chi=198^{\circ}\right)$ at a given epoch. The short ridge-lines fall on top of the long ones and do not show symmetry around the axis as they display, at most, half of the longest-observed wavelength, which determines the jet axis. In contrast, Figure 13 shows the location of the ridge-line for different epochs at a given frequency $(15 \mathrm{GHz})$, using the position angle of the jet at this frequency $\left(\chi_{15}=210^{\circ}\right)$ for the sake of clarity and to facilitate the detection of wavelengths in this plot.

At $1.6 \mathrm{GHz}$, the ridge-line of the jet is dominated by a long wavelength oscillation of $\simeq 80$ mas (Fig.12). A small scale modulation of 10 mas is also observed in the inner region $(<6$ mas to the core, as indicated in the bottom right panel of Fig. 12). $\mathrm{A} \simeq 20$ mas structure is observed in the inner 40 mas at 2 and $5 \mathrm{GHz}$ (e.g., left bottom panel in Fig. 12). Farther downstream, a high-amplitude ( $\simeq 4$ mas) structure with wavelength $\simeq 40$ mas is the most remarkable feature (e.g., upper right panel in Fig. 12). There is an even shorter, small-amplitude wave, with wavelength 5 mas, observed at 8 and $15 \mathrm{GHz}$ in the inner 15 mas, followed by another with wavelength 7-8 mas between 20 and 30 mas (indicated in the bottom right panel of Fig. (12). Beyond 30 mas the data for the higher frequencies have low signal-to-noise. Figure 14 shows the ridge-lines at $1.6 \mathrm{GHz}$ at different epochs (1997, 2003 and 2008). Although the main structures are very similar, the long wavelength is not so clearly observed in the last epochs and short-wavelengths seem to dominate the structure at larger distances from the core.

The ridge-line at $1.6 \mathrm{GHz}$ shows two peaks starting at 80 mas from the core in the first epoch (Fig. 12, upperleft panel). The first peak does not appear in the next epochs (see Fig. 14). In addition, there is a low signalto-noise in this region. This leads to doubts as to the real nature of the first peak. The second increase in the amplitude (120-140 mas) is present in all three epochs.

From the radio maps, we see that the jet emission drops when the ridge-line perturbation rises in the $\mathrm{x}-\mathrm{y}$ plot at $1.6 \mathrm{GHz}$. This can be interpreted as the jet veering slightly away from the line of sight. Moreover, the jet at higher frequencies is seen only up to $30-40$ mas, i.e., exactly where this effect should be expected if the ridge-line veers away from the observer. This deviation is, nevertheless, very small because the amplitude of the perturbation is small compared to the axial wave-length ( $A \sim 4$ mas and $\lambda_{\text {obs }} \simeq 80$ mas, see Fig. (12) .

\subsection{Wave speeds}

We have tried to measure the speed of the waves, both transversally and axially. To obtain a transversal motion we take fixed points in the $x_{o b}$ coordinate, defined by Equation 1 and Figure 11, and derive the displacements in the $y_{o b}$ coordinate between two epochs. By doing this, we would expect an oscillation in the transversal velocities if the apparent sinusoidal wave is moving spatially along the $x_{o b}$-axis or if a standing sinusoidal wave pattern is growing temporally in amplitude. The first and second images in Figure 15 give the transverse velocities 

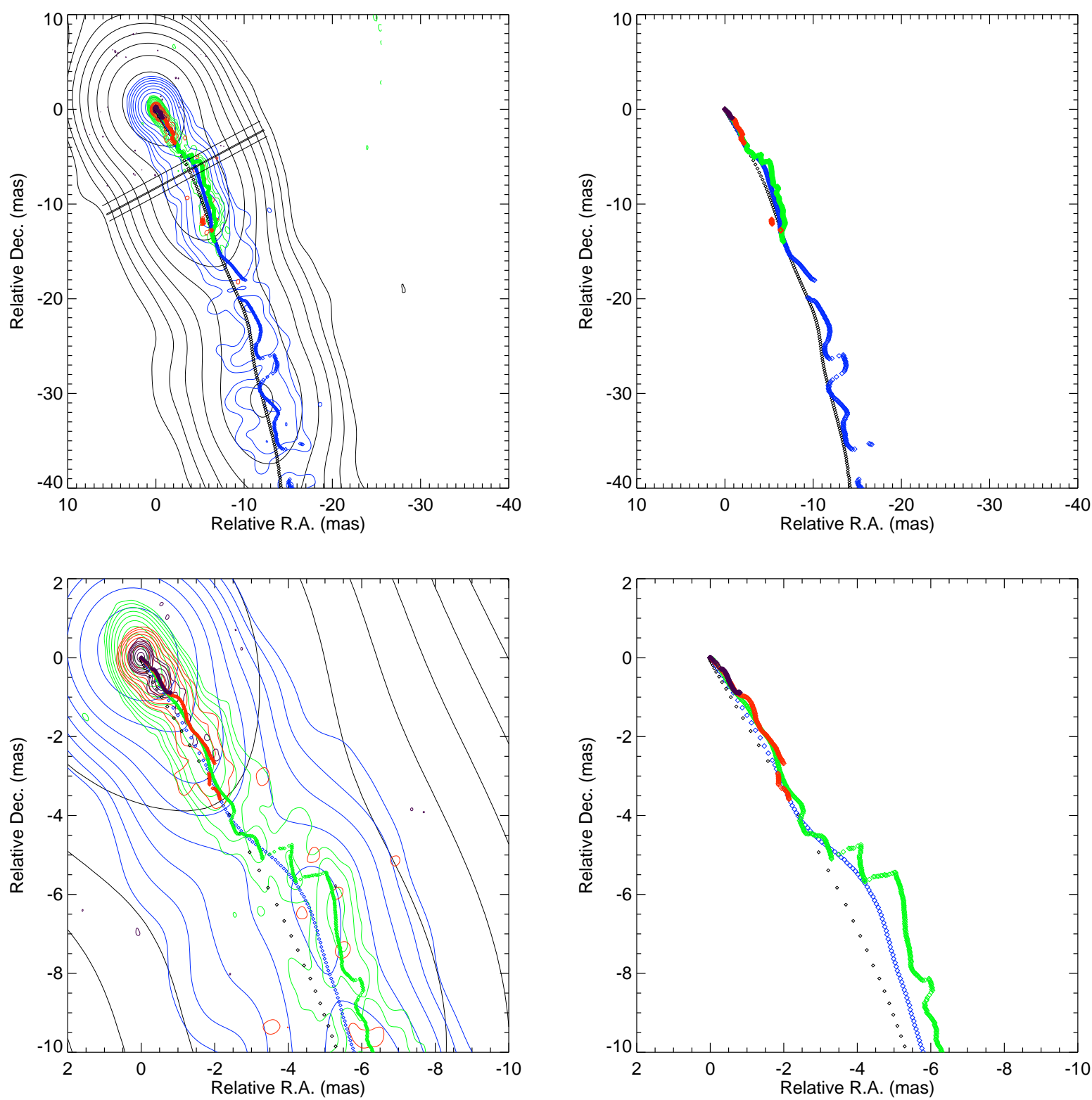

Fig. 7.- Radio maps at the 1.6 (black contours), 5 (blue contours), 15 (green contours), 22 (red contours) and 43 GHz (violet contours) of the jet in $0836+710$ in 2003 . The ridge-lines at the different frequencies are indicated by diamonds in the same colour as the contours and are shown without contours on the right. The black lines in the top-left panel indicate the locations of the cuts shown in Fig. 10 The bottom panels show an enlarged image of the inner region.

that are found using the difference in ridge-line position between $15 \mathrm{GHz}$ VLBA observations made in 2002 and 2003, and made in 2008 and 2009 (see Table 1). Figure 15 shows transversal velocities from the radio core out to about 4 mas. The images for this part of the jet in 2002 and 2009 can be found in the bottom leftmost and rightmost panels in Figure2. In Figure 15] we see that the transversal velocity is zero at the radio core since here it is assumed that the radio core is at a fixed position. In this regard note that the oscillation in transversal velocities is not symmetric around zero and we will return to a more detailed discussion of this and other transversal velocity issues in Section 6. The oscillation pattern could be produced by a growing standing wave or a moving wave. However, the velocities obtained are larger than $c$ and for a standing wave would imply amplitude growth at greater than light-speed and thus not possible. On the other hand, it is possible to find a transverse speed greater than $c$ for a moving wave and we will show under what conditions in the next sections. Additionally, we observe an identical region of the jet that the oscillation pattern in transversal velocity has shifted between the epoch pairs along the $x_{o b}$-axis by $\sim 0.4$ mas and includes only negative values for the transversal velocity. This shift indicates motion of the pattern along the $x_{o b}$-axis that would not occur for a temporally growing standing wave. We will return to the reasons for the lack of similar maxima in the positive and negative velocities in Section 6. However, the errors in the ridge-line position are usually larger than the equivalent distance travelled by light in the interval between epochs. This results in values larger than $c$ even if the true values are less than 


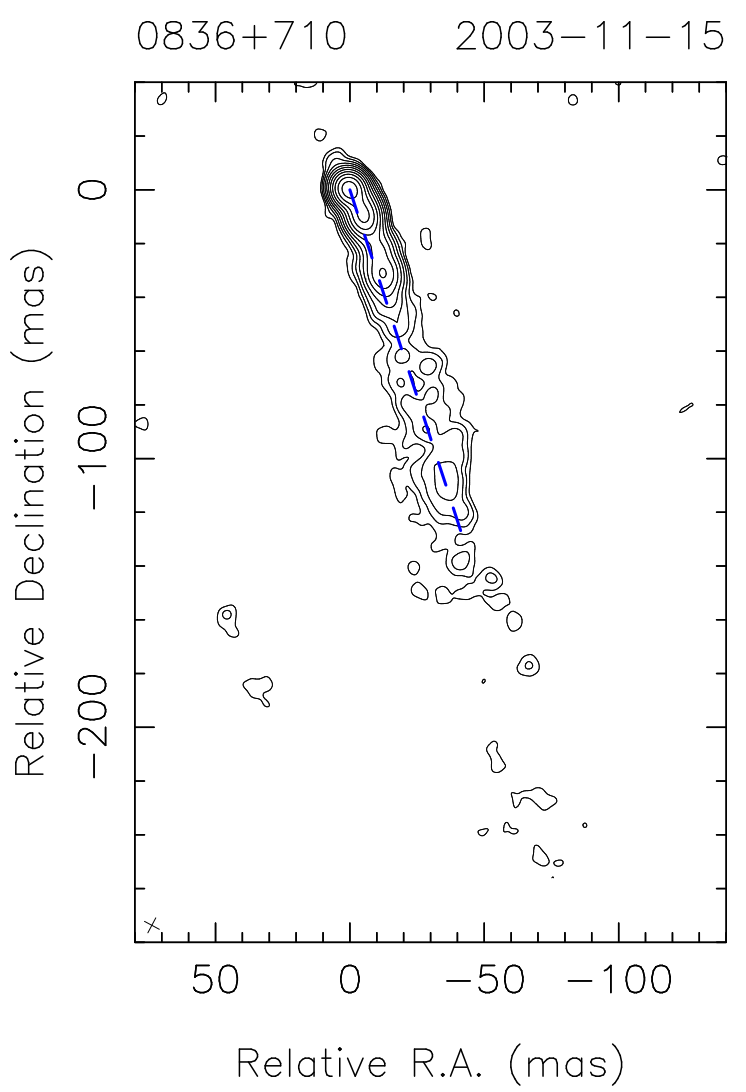

Fig. 8. - 1.6 GHz map of the jet in 2003. The dashed-blue line indicates the position angle of the jet axis with respect to the positive Relative Declination. $\left(198^{\circ}\right)$.

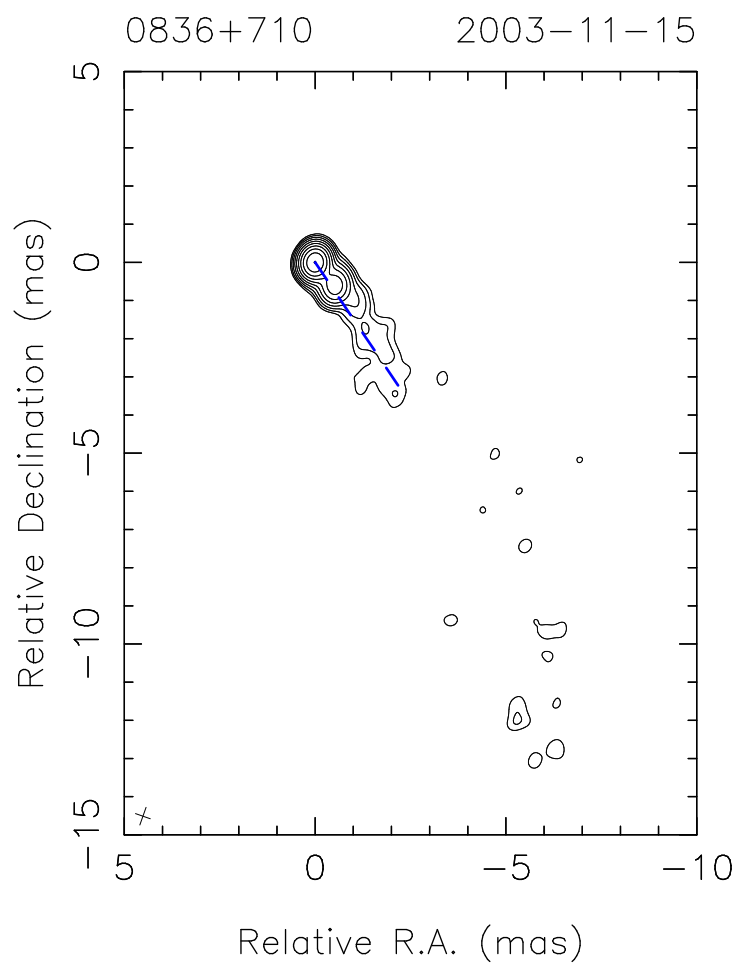

FIG. 9. - $22 \mathrm{GHz}$ map of the jet in 2003 . The dashed-blue line indicates the position angle of the jet axis with respect to the positive Relative Declination. $\left(214^{\circ}\right)$.

c. Actually, we have found that the maxima in transver- sal velocity are larger for shorter time-intervals between epochs, where less real motion is expected and differences in positions are dominated by errors. Thus, we cannot conclusively rule out a temporally growing standing wave based on the transverse velocities in Figure 15. but examination of the differences between the 2002 and 2009 $15 \mathrm{GHz}$ panels in Figure 2 allows us to conclude that a moving wave pattern is the most likely cause of the transversal velocities.

It is also possible to compute the axial speed, using fixed $y_{o b}$ position and computing the difference in the projected axial coordinate between epochs. We would expect a constant value for this speed if the wave is moving. In fact we do not obtain a constant velocity. Although the evolution of the ridge-lines at $15 \mathrm{GHz}$ (see Fig. 13) shows that the small peak at 4 mas in the left top panel seems to move to the right with time, no regular velocity pattern has been found in relation with this motion. The reason for this might be errors in determining the axial locations due to blending of the axial and the second transversal spatial coordinates on the plane of the sky. In addition, whereas in the case of only one dominant wavelength, the measure could be easy, the superposition of the various different wavelengths with different potential speeds would lead to variability in displacements from epoch to epoch. In the next sections, we provide the equations that couple the transverse and axial speeds with the amplitude and wavelengths. However, a detailed derivation of these equations will be presented elsewhere (Perucho et al., in preparation).

\section{FROM OBSERVATIONS TO THEORY}

\subsection{Observed and intrinsic structures}

Past work has shown that oscillations like that observed in $0836+710$ can be produced by threedimensional helices developing in a relativistic outflow. In this case the position of the ridge-line will trace the accompanying helically twisted pressure maximum along the jet, e.g., the evidence presented in Section 3 . In order to model these structures we have to take into account projection and relativistic effects due to the possible propagation of the waves with a high velocity.

From Figure 11, we can see that the coordinate $y_{o b}$ is not affected by projection or light travel time-delay and coincides with the corresponding coordinate of the intrinsic helix $y_{h}$. However, coordinate $x_{o b}$ is affected by both projection and light travel-time effects and depends on both the transversal coordinate $x_{h}$ and on the position $z_{h}$ along the axis of the helix and the jet itself.

The observed helical wavelength is related to the intrinsic on 6 via the viewing angle, $\alpha$, and the intrinsic wave speed, $\beta_{w}$, following 7

$$
\lambda_{o b s}=\lambda_{i n t} \frac{\sin \alpha}{\left(1-\beta_{w} \cos \alpha\right)},
$$

The intrinsic equations describing a helix moving along

${ }^{6}$ We will call intrinsic a perpendicular position (viewing angle of $90^{\circ}$ ) of an observer at rest with respect to the jet flow.

7 cf. Perucho \& Lobanov (2007), where cosmological effects were mistakenly considered: the redshift effect in the frequency also affects the measured velocities, but not the measured wavelengths. 

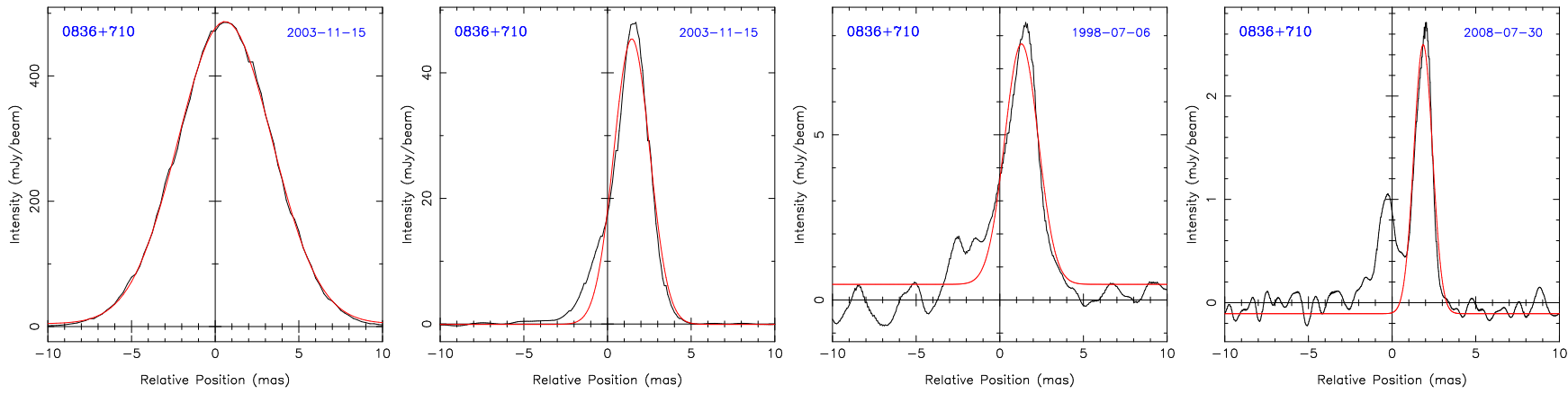

FIG. 10.- Profiles and Gaussian fits for 1.6 and $5 \mathrm{GHz}$ (2003 epochs), $8 \mathrm{GHz}$ (1998) and $15 \mathrm{GHz}$ (2006), from left to right. The location of the cuts is shown in the top-left panel of Fig. 7 (the first two panels here are taken from the epoch in that Figure, whereas the other two have been plotted together for the sake of clarity). In the left panel $(1.6 \mathrm{GHz})$, the cut is done 7.50 mas from the core along the axis $\left(\mathrm{PA}=-153.4^{\circ}\right)$, the peak intensity is $482.5 \mathrm{mJy} / \mathrm{bm}$, the peak relative position is 0.591 mas, and the FWHM of the Gaussian is 6.630 mas. In the second panel from the left $(5 \mathrm{GHz})$, the cut is done 7.40 mas from the core along the axis $\left(\mathrm{PA}=-153.4^{\circ}\right)$, the peak intensity is $45.5 \mathrm{mJy} / \mathrm{bm}$, the peak relative position is $1.454 \mathrm{mas}$, and the FWHM of the Gaussian is $2.533 \mathrm{mas}$. In the third panel (8 GHz), the cut is done 6.40 mas from the core along the axis $\left(\mathrm{PA}=-153.4^{\circ}\right)$, the peak intensity is $7.3 \mathrm{mJy} / \mathrm{bm}$, the peak relative position is 1.298 mas, and the FWHM of the Gaussian is 2.362 mas. In the right panel $(15 \mathrm{GHz})$, the cut is done 8.20 mas from the core along the axis $\left(\mathrm{PA}=-153.4^{\circ}\right)$, the peak intensity is $2.6 \mathrm{mJy} / \mathrm{bm}$, the primary peak relative position is 1.859 mas, and the FWHM of the Gaussian is 1.31 mas.

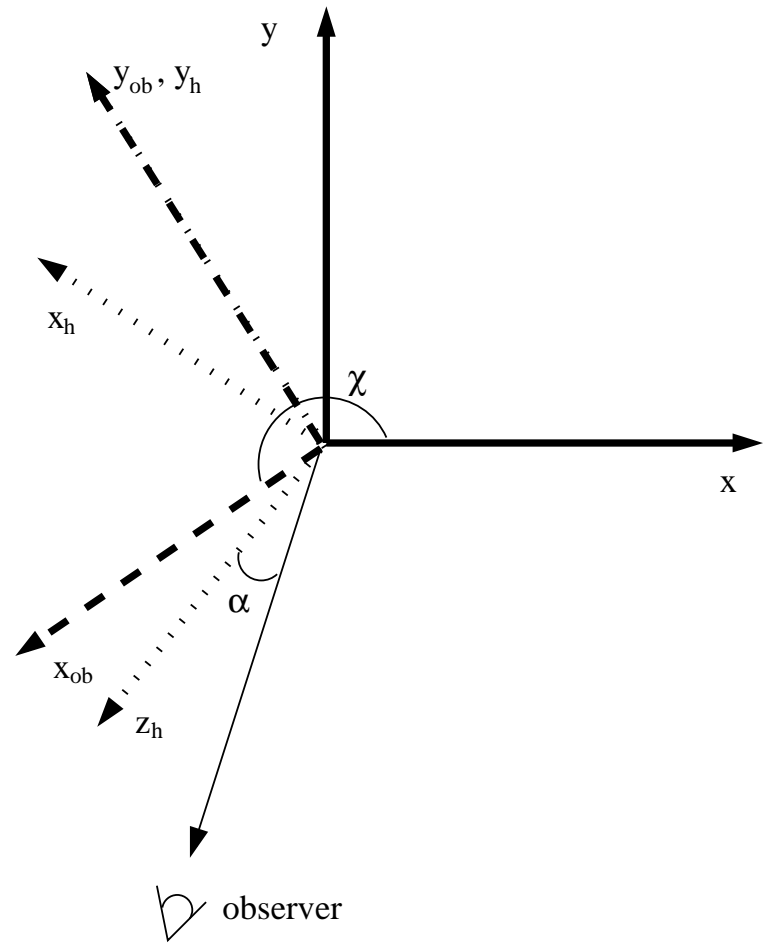

FIG. 11.- Coordinate systems used in the text. The intrinsic coordinates of the helix are $x_{h}, y_{h}$ and $z_{h}$. The observed coordinates are $x_{o b}$ and $y_{o b} . x, y, x_{o b}$, and the axis including $y_{o b}$ and $y_{h}$ fall on the plane of the sky. Note that this image is mirrored with respect to the radio maps in the paper.

the $z_{h}$ axis with speed $v_{w}$ are:

$$
\begin{array}{r}
x_{h}=A_{0} e^{z_{h} / \lambda_{i}} \cos \left(\frac{2 \pi\left(z_{h}-v_{w} t\right)}{\lambda}+\varphi_{0}\right) \\
y_{h}=A_{0} e^{z_{h} / \lambda_{i}} \sin \left(\frac{2 \pi\left(z_{h}-v_{w} t\right)}{\lambda}+\varphi_{0}\right)
\end{array}
$$

where $A_{0}$ is the initial amplitude of the helix, $\lambda_{i}$ is the growth length of the helix if it couples to an instability, $\lambda$ is the wavelength, and $\varphi_{0}$ is the phase. These four variables plus the wave velocity are the unknowns that determine the structure of the wave. Finally, $t$ is the time, and $z_{h}$ is the axial coordinate. This helix is pro- jected onto the sky plane $\left(x_{o b}, y_{o b}\right)$ where the helical axis coordinate, $z_{h}$, and $x_{h}$ are to be projected onto the sky plane $\mathrm{x}$-axis and the helical axis makes an angle, $\alpha$, with respect to the line of sight (which lies in the $x_{h}, z_{h}$ plane). The relation between the observed $\left(x_{o b}, y_{o b}\right)$ and intrinsic $\left(x_{h}, y_{h}, z_{h}\right)$ coordinates is the following (Perucho et al., in preparation):

$$
\begin{array}{r}
x_{o b}=x_{h}\left(z_{h}\right)\left[\cos \alpha-\left(\frac{\sin ^{2} \alpha}{1-\beta_{w} \cos \alpha}\right) \beta_{w}\right]+ \\
z_{h} \frac{\sin \alpha}{\left(1-\beta_{w} \cos \alpha\right)} \\
y_{o b}=y_{h} .
\end{array}
$$

which, in the absence of motion reduces to:

$$
\begin{array}{r}
x_{o b}=x_{h} \cos \alpha+z_{h} \sin \alpha \\
y_{o b}=y_{h},
\end{array}
$$

The observed ridge-line points could then be fitted to a helix by means of a minimizing $\chi^{2}$ method, using the above equations. However, Equation (4) embeds a key degeneracy in the problem: the observed wavelength is a combination of the wave velocity and the intrinsic wavelength. Thus, there are different combinations of both parameters that may result, for a given viewing angle, in the observed wavelength. In order to uniquely identify the intrinsic wavelengths it is necessary to measure the wave speed of the different structures. A simple way to do this would be to measure motions in the ridgeline between epochs by following visible features such as maxima or minima and relate those to intrinsic motions. However, we have seen in Section 3.5 how difficult this may be with the present accuracy of the VLBI observations. Thus, we can only find limits to the parameters implied by the structure.

An additional complication arises when more than one periodical structure is found in a jet. In this case each periodical structure has to be independently fitted if it propagates with different speed. The reason is that the relativistic and projection effects, which depend on the propagation velocity, determine the position of the displacements along the observed axis following Equation (4), which makes independent parametrization nec- 

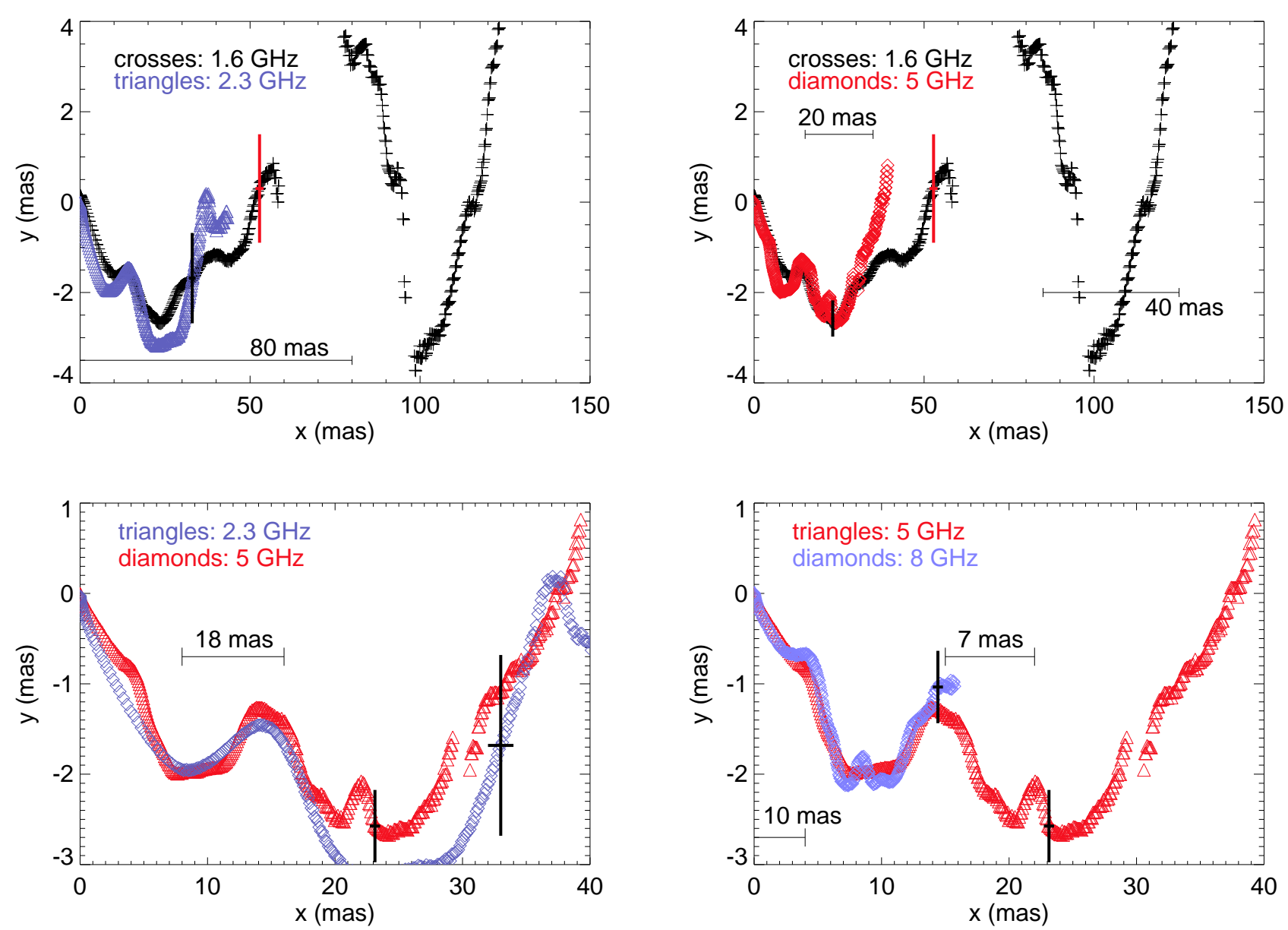

FIG. 12.- Transverse displacement of the jet ridge-line around the jet axis at several frequencies in 1997. 1.6, 5 and 8 GHz images were obtained with VLBA data, whereas the $2 \mathrm{GHz}$ one was obtained with Global VLBI. We indicate the wavelengths by the observed sizes between maxima or minima (half a wavelength in the case of the 10 and 18 mas oscillations). The black or red crosses show the error bars (one fifth of the beam size in the direction of the displacements), at randomly selected points, to be applied to each data point in the ridge-line at a given frequency. The errors are not shown at all points for the sake of clarity.

essary. Once the velocity and intrinsic wavelength of each wave are known, their contributions to the ridge-line displacement, $y_{o b}$ in Equation 4, at a given location, $x_{o b}$, have to be added and then compared to the observed ones.

A more detailed description of the modeling of relativistic helices will be published elsewhere (Perucho et al., in preparation).

\section{JET PROPERTIES DERIVED FROM THE OBSERVATIONS}

\subsection{The 40 mas wave}

In all three ridge-lines observed at $1.6 \mathrm{GHz}$, a 40 mas wavelength between $x \simeq 80-120$ mas (see Fig. 4), indicates a shortening of the wavelength dominating the shape of the ridge-line and deserves some discussion. This structure can be explained in one of the following three ways: a) the amplitude of a shorter wavelength increases to larger than that of a longer one, b) the angle to the line of sight decreases and the long wavelength is observed with a shorter wavelength, or c) the longer wavelength is decelerated. Options $b$ and $c$ follow from Equation 2 that relates the observed and intrinsic wavelengths, taking into account projection and relativistic effects.

We discuss here the different possibilities:

- a: The longer initial wavelength could be due to a forced change in the jet direction that due to its low frequency couples to a long wavelength slowly growing $\mathrm{KH}$ mode. The shorter wavelength is the result of a faster growing, possibly resonant, $\mathrm{KH}$ mode, whose amplitude grows sufficiently rapidly to become larger than that of the forced oscillation. Thus, this is a viable option.

- b: We can find the required decrease in the angle to the line of sight for the 80 mas wavelength to change to the 40 mas wavelength using Equation 2, and keeping the intrinsic wavelength and wave speed constant. For small viewing angles Equation 2 becomes $1-\beta_{w, 1} \cos \alpha_{1} \simeq 1-\beta_{w, 2} \cos \alpha_{2}$

8 Considering that the changes discussed occur already at large de-projected distances from the core and that there are evidences of $\mathrm{KH}$ instability to dominate at distances larger than a few parsecs (see, e.g., Hardee 2006, 2011; Perucho 2011), we will restrict the discussion to this kind of instability. 

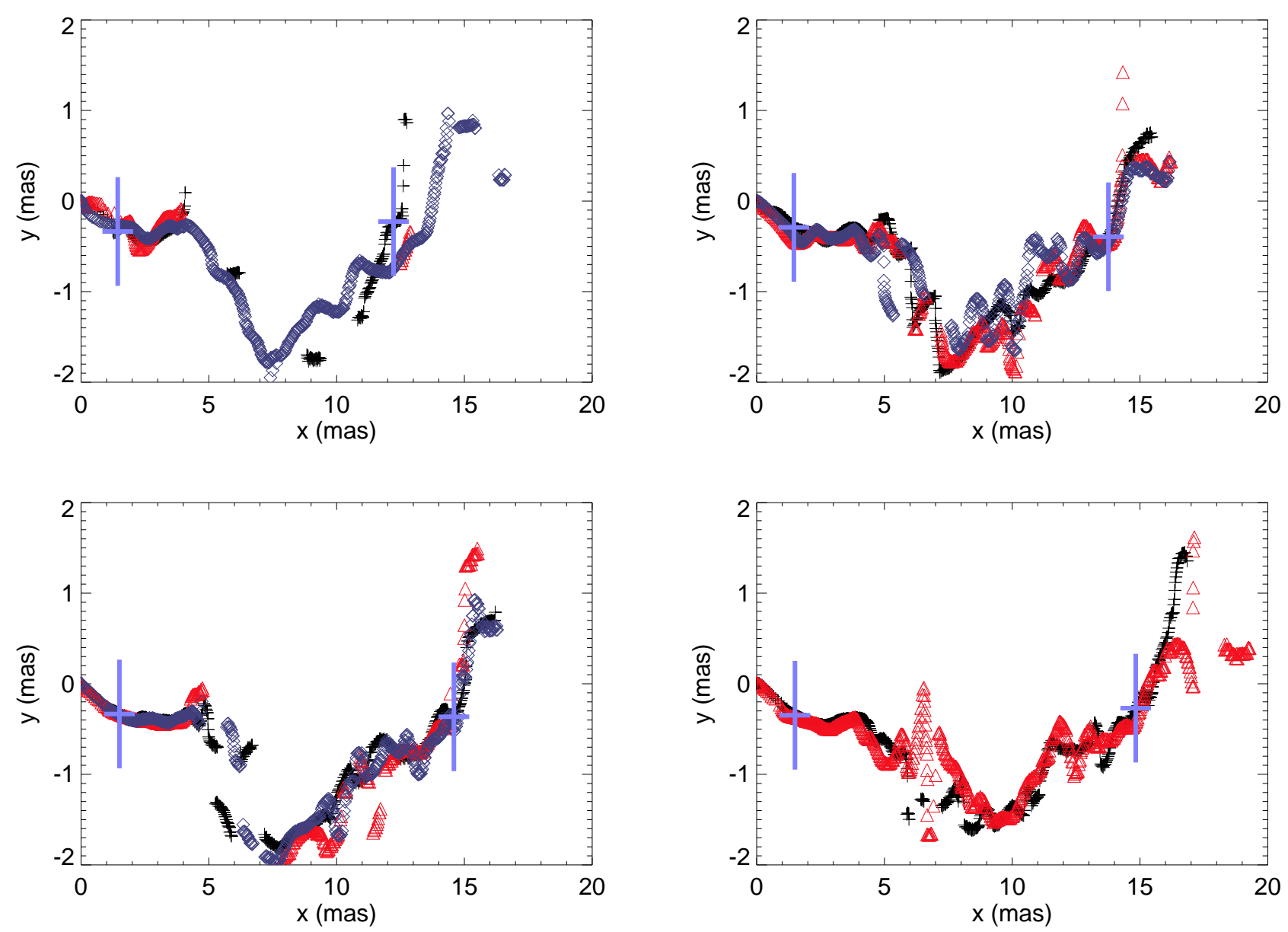

Fig. 13. - Transverse displacement of the jet ridge-line at $15 \mathrm{GHz}$ and different epochs around the position angle of the jet at this frequency, ranging from 1998 to 2009. In the upper left panel, black crosses represent the ridge-line from March 1998, red triangles from June 2000 and blue diamonds from June 2002. In the upper right panel, black crosses represent the ridge-line from March 2003, red triangles from September 2004 and blue diamonds from May 2005. In the lower left panel, black crosses represent the ridge-line from October 2005, red triangles from September 2006 and blue diamonds from August 2007. In the lower right panel, black crosses represent the ridge-line from July 2008 epoch and red triangles from May 2009. As in Fig. 12 the blue crosses show the error bars (one fifth of the beam size in the direction of the displacements), at randomly selected points, to be applied to each data point in the ridge-lines. The errors are not shown at all points for the sake of clarity.

with an error of $<10 \%$, and:

$$
\frac{\sin \alpha_{1}}{\sin \alpha_{2}} \simeq \frac{\lambda_{o b s, 1}}{\lambda_{o b s_{2}}} \simeq 2 .
$$

This means that $\alpha_{2} \simeq \alpha_{1} / 2$, and a decrease in the angle to the line of sight down to one half of its original value is needed. This could be produced by a change in the conditions of the ambient medium when the jet exits the ISM of the galaxy. However, this would imply the presence of a standing shock where the jet changes direction. Such a shock is not observed and there is no hint that the jet direction is appreciably changed. Thus, this option is unlikely.

- c: A decrease in the velocity of propagation of the 80 mas wave could also lead to the observed wavelength decrease. It is well-known that the perturbations propagating in a flow cannot have velocities larger than that of the underlying fluid. For example, if the flow decelerates, the wave would slow as well. Now using Equation 2 and keeping the intrin- sic wavelength and the viewing angle constant we have:

$$
\frac{\left(1-\beta_{w, 2} \cos \alpha\right)}{\left(1-\beta_{w, 1} \cos \alpha\right)}=\frac{\lambda_{o b s, 1}}{\lambda_{o b s, 2}} \simeq 2,
$$

which should be solved for $\beta_{w, 2}$. For example, taking $\beta_{w, 1}=0.9$ for the inner region, $\alpha=3^{\circ}$, $\lambda_{o b s, 1}=80$ mas and $\lambda_{o b s, 2}=40$ mas, we obtain $\beta_{w, 2}=0.8$, whereas for $\beta_{w, 1}=0.6$ we get $\beta_{w, 2}=0.2$. This option allows us to place constraints on $\beta_{w, 1}$ using $\lambda_{o b s, 1}\left(1-\beta_{w, 1} \cos \alpha\right)<\lambda_{o b s, 2}$, to obtain $\beta_{w, 1}>0.5$. It is known that a flow can be decelerated when a helical perturbation grows to nonlinear values and disrupts the collimated flow resulting in conversion of kinetic into thermal energy (see e.g., Perucho et al. 2005, 2010). The uncollimated structure of the $0836+710$ jet at kiloparsec scales indicates that this is a viable option.

Figure 14 showed that the long wavelength is not clearly observed in the later epochs at $1.6 \mathrm{GHz}$ and shortwavelengths seem to dominate the structure at larger dis- 

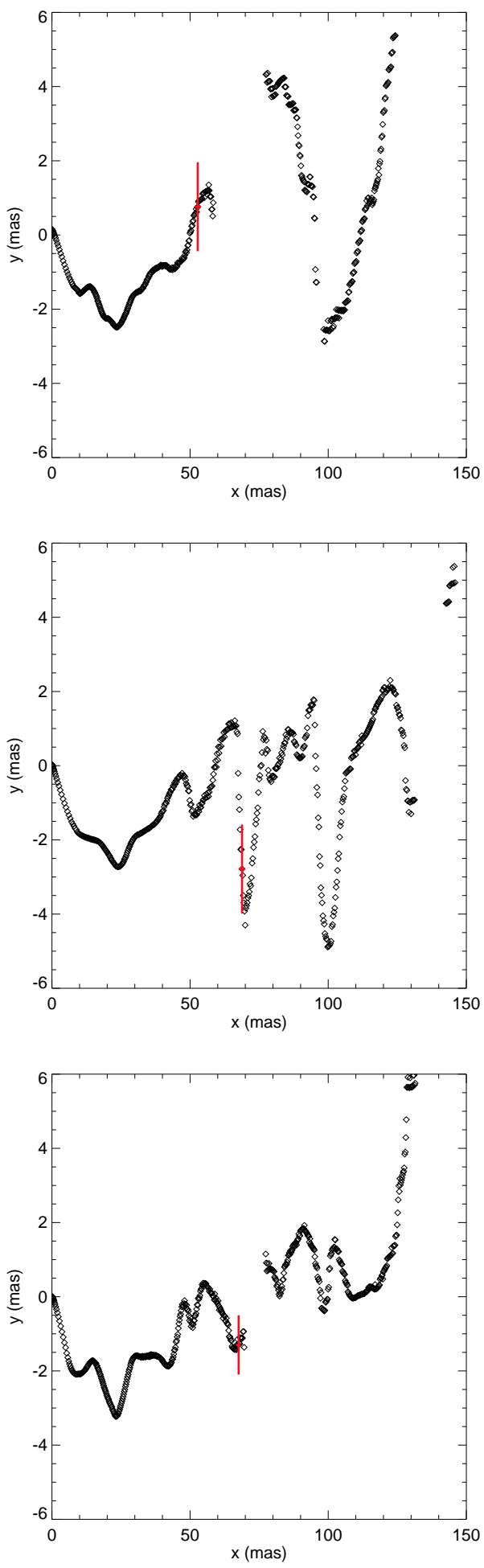

FIG. 14.- Rotated ridge-lines obtained for $1.6 \mathrm{GHz}$ in 1997 (top), 2003 (center) and 2008 (bottom). As in Fig. 12 the red crosses show the error bars (one fifth of the beam size in the direction of the displacements), at randomly selected points, to be applied to each data point in the ridge-lines. The errors are not represented for all points for the sake of clarity.

tances from the core at the later epochs. It seems likely that some combination of options $a$ and $c$ are favored by the observations.

\subsection{On the presence of a shear-layer in the jet}

Perucho \& Lobanov (2007) presented results that pointed towards the jet in $0836+710$ having a significant velocity shear layer. For this study, the authors used as a set of jet parameters those resulting from a previous work by Lobanov et al. (1998): Lorentz factor $\gamma=11$, Mach number $M_{j}=6$, and jet/ambient density ratio $\rho_{j} / \rho_{a}=0.04$. They studied the different characteristic wavelengths growing in a jet with a thin shear-layer $(\simeq 10 \%$ of the jet radius $)$, and a thick one $(\simeq$ $60 \%$ of the jet radius). The result showed that the observed wavelengths in the jet structure could be better explained transversally stratified jet. A mistake in the equation used in that work (Perucho \& Lobanov 2011) led to misidentification of the modes in the original work, but the conclusions were shown to remain valid.

In the present work, we have been able to accurately measure the wavelength of the longest mode to be closer to 80 mas than to 100 mas. A correct treatment reduces the values of the wavelengths obtained in Table 1 of Perucho \& Lobanov (2007) by a factor of $\simeq 3$. This implies that their solutions both with and without a shear layer cannot explain the observed structures, reported to be 100 mas and 7.7 mas (Lobanov et al. 1998), with the jet parameters used to obtain the results shown in Figure 1 of Perucho \& Lobanov (2007). We reproduce here the solutions for the sheared jet in Fig. 16.

Taking 80 mas for the wavelength, $3^{\circ}$ for the viewing angle, and that the velocity of this long wave has to be $\beta_{w}>0.5$ (see Section 5.1), we obtain an upper limit to the intrinsic wavelength using Equation 2 of

$$
\lambda_{\text {int }, 80 \text { mas }}<\left(\frac{1-0.5 \cos 3^{\circ}}{\sin 3^{\circ}}\right) 80 \text { mas }=765 \text { mas. }
$$

In terms of the solutions presented in Perucho \& Lobanov (2007), this is $\lambda_{\text {int.80 mas }}<45 R_{\mathrm{j}}$, with $R_{\mathrm{j}}=17$ mas (Perucho \& Lobanov 2007). Now the maximum growth rate of the surface mode occurs at $160 R_{\mathrm{j}}$ for the sheared jet and at $105 R_{\mathrm{j}}$ for the vortex sheet jet, which is clearly different from the obtained intrinsic wavelength. For the sheared jet, the maximum growth rate of the first body mode is at the frequency $\omega \simeq 0.1 c / R_{j}$ and $\lambda \simeq 42 R_{\mathrm{j}}$. Taking into account that its velocity is $\simeq v_{w} \simeq 0.5 c$ (computed as $\left.\simeq R e(\omega / k)\right)$, we obtain a value very close to $\lambda_{i n t, 80 \mathrm{mas}}$. We note that the maximum growth rate of the first body mode for the vortex sheet solution is at $\lambda \simeq 21 R_{\mathrm{j}}$, with $v_{w} \simeq 0.6 \mathrm{c}$, farther away from the required properties to explain the observed wavelength. While the first body mode is not the fastest growing one in the solution, it could be triggered and generate large deformations in the jet, which are not expected to be caused by high-order body modes (Perucho et al. 2005, 2007, 2010). We note that this interpretation is very dependent on the measured jet radius.

At the same triggering frequency $\left(\omega \simeq 0.1 c / R_{j}\right)$ in the sheared case, high-order body modes could produce the $7-8$ mas wavelengths (identified with the 7.7 mas wavelengths studied in Perucho \& Lobanov 2007). This wavelength could really be $\simeq 10$ mas, resulting an intrinsic $\lambda_{\text {int,10 mas }} \simeq 9 R_{\mathrm{j}}$, obtained using $3^{\circ}$ viewing angle and $v_{w} \simeq 0.2 c(\simeq \operatorname{Re}(\omega / k))$. The fact that both growing modes would be triggered at the same frequency sim- 

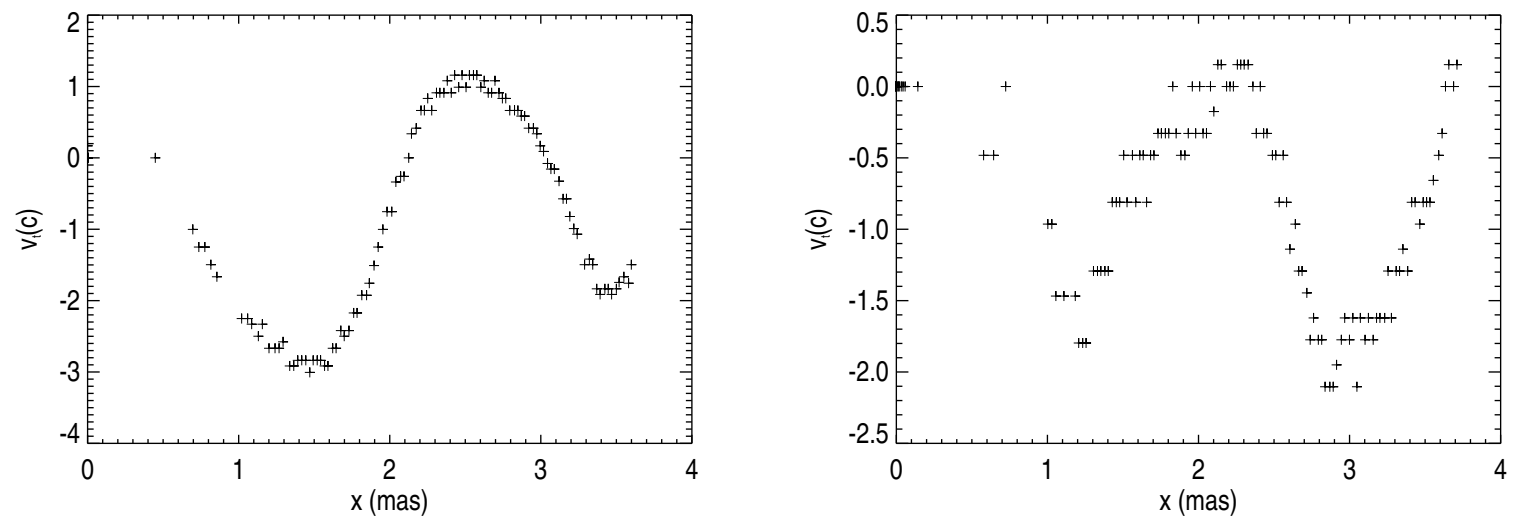

Fig. 15.- Transversal velocities measured from 15 GHz VLBA data between epochs 2002-2003 and 2008-2009, respectively.
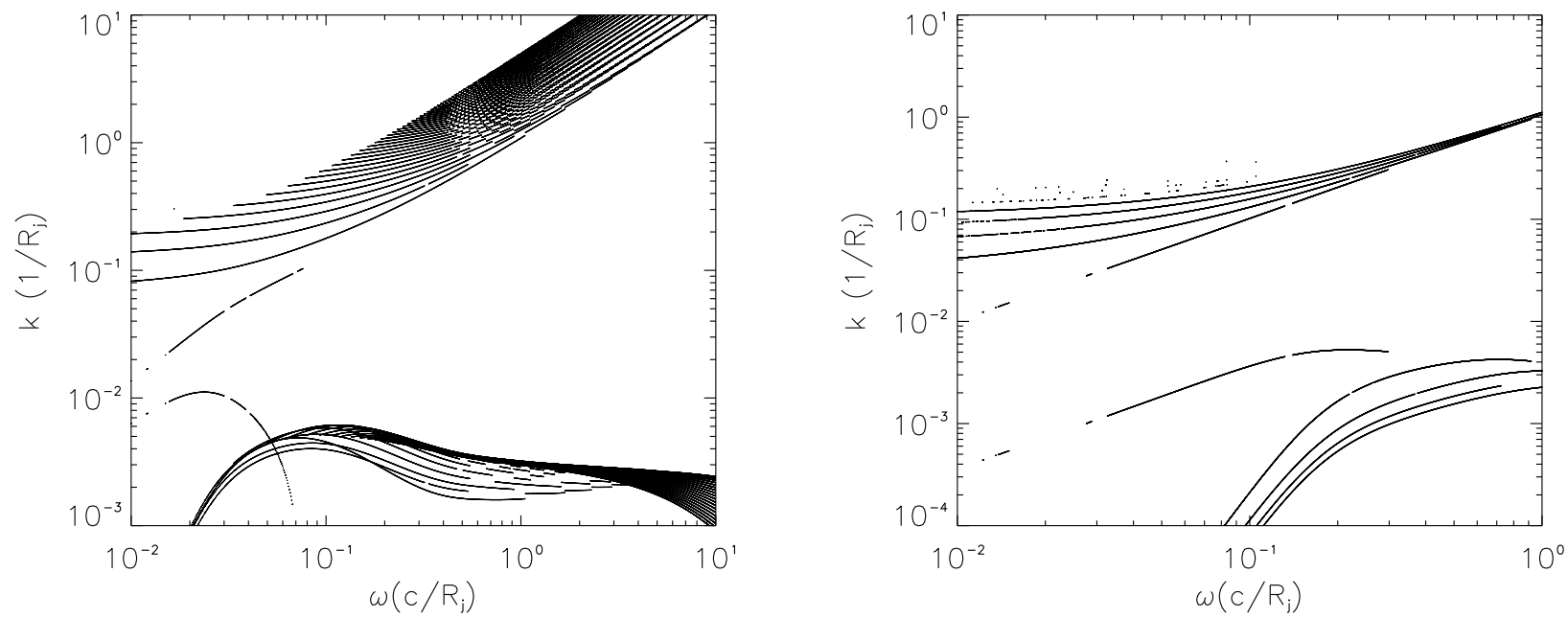

FIG. 16.- Solutions of the linear problem of KH instability for a sheared jet (see Perucho \& Lobanov 2007, for details). The left panel shows the real (upper curves) and imaginary (lower curves) parts wavenumber with wave-frequency, whereas the right panel shows the wave velocity $(\operatorname{Re}(\omega / k))$ with wave-frequency.

plifies the nature of the driving mechanism or mechanisms to a single one. In this case, the small wavelengths can be triggered as harmonics of the longest excited one (Perucho et al. 2005). The new resulting frequency is a factor of 4 larger than that given in PL07 for the longest wavelength. Thus, the driving period would be now $T_{d r} \simeq 1.4 \times 10^{7} \mathrm{yrs}$, and similar to the one given by Hardee et al. (1994) for 3C 449.

The helical jet structure and appearance is assumed to be due to $\mathrm{KH}$ instability as in Hardee (2000, 2003) and Hardee et al. (2005). In these works, it is assumed that the velocity shear layer is very thin compared to the jet radius and wavelength (vortex sheet approximation). We note that long wavelengths should be insensitive to a modest shear layer so a vortex sheet long wavelength analytical approximation would be good enough to describe the behavior at long wavelengths. An alternative interpretation to the one in Perucho \& Lobanov (2007, 2011) is that the longest wavelength of $\lambda_{h}^{o b} \sim 80$ mas is in the long wavelength and low frequency limit of the $\mathrm{KH}$ instability spectrum: $\omega<<<\omega^{*}$, with $\omega^{*}$ being the frequency of the maximum growth rate. Also, we might assume that the intermediate wavelength that appears to increase from about $\lambda_{h}^{o b} \sim 10-12$ mas $\left(x_{o b} \sim 0-5\right.$ mas $)$ to $\lambda_{h}^{o b} \sim 20-24$ mas $\left(x_{o b} \sim 5-15\right.$ mas $)$ to $\lambda_{h}^{o b} \sim 40-48$ mas $\left(x_{o b} \sim 15-35\right.$ mas $)$ at the different apparent distances along the jet are each operating at about the local resonant wavelength and frequency, i.e., $\omega \sim \omega^{*} \propto a_{e x} / r_{j}$. Finally, the shortest wavelengths $\lambda_{h}^{o b} \sim 4.5$ mas $\left(x_{o b} \sim 5-15\right.$ mas $)$ and $\lambda_{h}^{o b} \sim 7-8$ mas $\left(x_{o b} \sim 20-30\right.$ mas $)$ might be assumed to be a body or shear layer mode operating at the appropriate resonant frequency and wavelength for that mode. This would require the jet having different parameters from those obtained in Lobanov et al. (1998).

As we see, different interpretations remain possible: vortex-sheet approximation for the longest wavelengths or changes in the jet width or in the viewing angle. In addition, there are still uncertainties such as the identification in Perucho \& Lobanov (2011) of the longest wavelength with a first body helical mode, instead of the more disruptive, surface mode at the small frequency limit. Overall, any results of this kind are subject to sources of error associated with the measure of the jet radius, the thickness of the shear-layer, the viewing angle or the 
parameters used for the jet.

\section{SUPERLUMINAL TRANSVERSAL MOTION AND} NATURE OF THE CORE

There exist different examples of jets showing apparent superluminal transversal motion (see, e.g., Agudo et al. 2007, 2010, 2011, the cases of NRAO 150 and OJ 287). We also observe this kind of motion for the ridge-line of $0836+710$ at $43 \mathrm{GHz}$. Within the context of the present work, there is a possible explanation for this phenomenon. We take into account the fact that the observations at high frequencies could be showing only the high pressure core of the jet along the ridge-line that we measure at lower frequencies (see Sections 2 and 4.1). This means that we may not observe the whole of the jet, but only the region around the high pressure maximum. As a result the radio core at $43 \mathrm{GHz}$ would itself oscillate transversely and axially over time and not be at a fixed position. If the radio core is associated with a reconfinement shock (Marscher et al. 2008), the presumed oscillations could well be the triggering point of the observed growing instabilities, except for the longest one, which could be driven by precession at the jet formation region.

Superluminal transversal velocities are possible for purely axial motion, a relatively large value for $\Delta y$ in time interval $\Delta t$, under some conditions (Perucho et al., in preparation). This very special situation may only occur for very small viewing angles when $v_{w}$ is close to the speed of light and we deal with high-frequency, short wavelength perturbations. There is a second possibility that we explore in the next paragraphs.

\subsection{Transversal shift of the apparent core}

One other possible reason for observed superluminal transversal motion lies in the mistaken assumption that the radio core remains at a fixed $\left(x_{o b}, y_{o b}\right)$ position. Since in our interpretation the radio core is taken as coincident with a pressure maximum inside the jet, we should expect an oscillation to take place in the radio core location, as the pressure maximum oscillates transversely in the plane of the sky and also oscillates axially with rate and amount depending on the properties of the helical wave (see Eq. 4). The oscillation should be small (much less than the jet radius) if only a result of an instability amplitude growth but other triggering mechanisms can be imagined that could lead to immediate helical amplitudes on the order of the jet radius. Therefore, when aligning the ridge-line (or any reference point) using the jet core as a fixed point at two different epochs, it is possible that the whole ridge-line or positions of fitted components are displaced by the same small amount as the core has moved in the transversal and axial direction. The effect of such artificial displacement will clearly affect any measure of velocities in the transversal direction. This oscillation has already been reported, using phase-referencing, in at least one object (M81, Martí-Vidal et al. 2011).

For the jet in $0836+710,1$ mas $\simeq 8.4 \mathrm{pc}$ (see Section 1), and a measured angular motion of only $0.012 \mathrm{mas} / \mathrm{yr}$ is equivalent to the speed of light. Thus, any small displacement of the ridge-line anchored at the core that is not corrected for possible core motion leads to incorrect transversal displacement at any point along the jet and can result in bogus measures of superluminal velocities in the transverse direction.

We have generated an artificial mode pattern of a helical wave with an intrinsic wavelength of $\lambda=2 R_{j}$, an initial amplitude of $A_{0}=0.2 R_{j}$, and a wave speed of $0.96 c$ in order to investigate the role of core shift in the measurement of transversal velocities. Figure 17 shows the resulting ridge-lines at two epochs separated by two years (left panel), and the resulting transversal velocities in units of the speed of light (right panel). Transversal velocities have been obtained by subtracting the vertical coordinate of the helix at the different times and dividing the result by the time interval, as we did for the observational data (see Fig. 15). With these parameters, the core moves transversally at an observed $0.006 \mathrm{mas} / \mathrm{yr}$. In this case, the intrinsic displacement of the core is respected, and we observe that all the velocities are subluminal with equal positive and negative maximum values. The displacement of the ridge-line along the axis is a consequence of the projection of the axial coordinate, $z_{h}$, and that perpendicular to the plane of the sky, $x_{h}$, (see Eq. 4).

In Figure 18 we re-plot the ridge-lines shown in Figure 17 but now with the cores at the two epochs forced to the same position at the origin. This artificial displacement of the core results in apparent superluminal motion. We have investigated the dependence of this effect with viewing angle, and axial wave velocity and amplitude. The effect does not depend on the viewing angle, as we measure transversal motions, but the transversal velocity does grow with wave amplitude and wave velocity. Note that positive and negative maximum transversal velocities are not the same and Figure 18 shows superluminal velocities very similar to those found between the 2008 and 2009 epochs shown in Figure 15. The similarities between the transversal velocities shown in Figure 18 and between the 2008 and 2009 epochs in Figure 15 provide indirect evidence for radio core displacement accompanying a moving helical wave pattern in $0836+710$. A direct observational test for core motion would consist of phase-referencing observations of nearby sources containing observed helical patterns (see, e.g., Martí-Vidal et al. 2011). In these cases small lateral angular displacements of the core, up to $0.012 \mathrm{mas} / \mathrm{yr}$ if the wave-velocity is close to the speed of light, might be directly detectable.

Core motion manages to explain the transversal velocities found in this work in an easy way and within the framework of our modeling of helical patterns. We note that the observed effect only requires very small displacements of the ridge-line in the core, well within the errors of our measurements. The caveats are that the wave velocity needs to be close to the speed of light and that the initial amplitude of the wave has to be a relatively large fraction of the wavelength, $10 \%$ in our example. However, from stability analysis we know that, at least in the case of $\mathrm{KH}$ modes, short wavelengths tend to move fast and can produce high pressures near to the jet surface. Thus, it should not be surprising to find such high pattern velocities when observing the spine of jets at high frequencies and close to the central engine. The proposed observational test can be used to verify this hypothesis. 

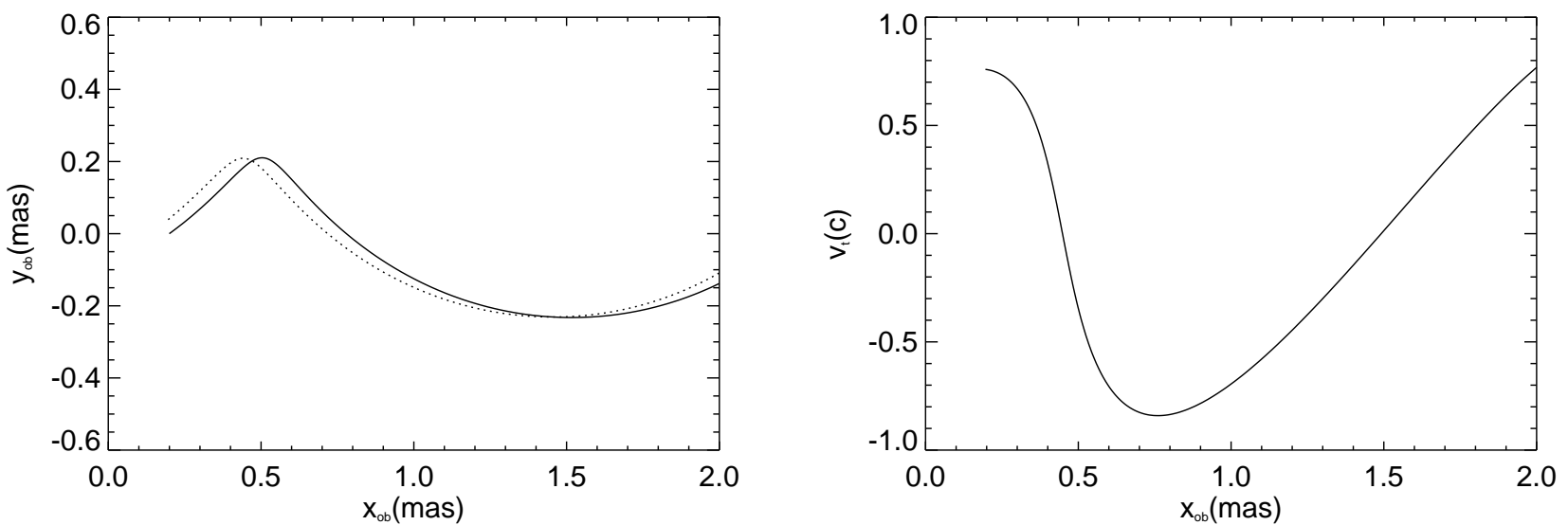

FIG. 17. - Absolute ridge-line position and motion at $3^{\circ}$ viewing angle without core position alignment. The left panel shows the ridgelines from the first epoch (solid line) and second epoch (dotted line) separated by a time interval of 2.11 years. The right panel shows the resulting transversal velocity versus the observed (projected) axial position.
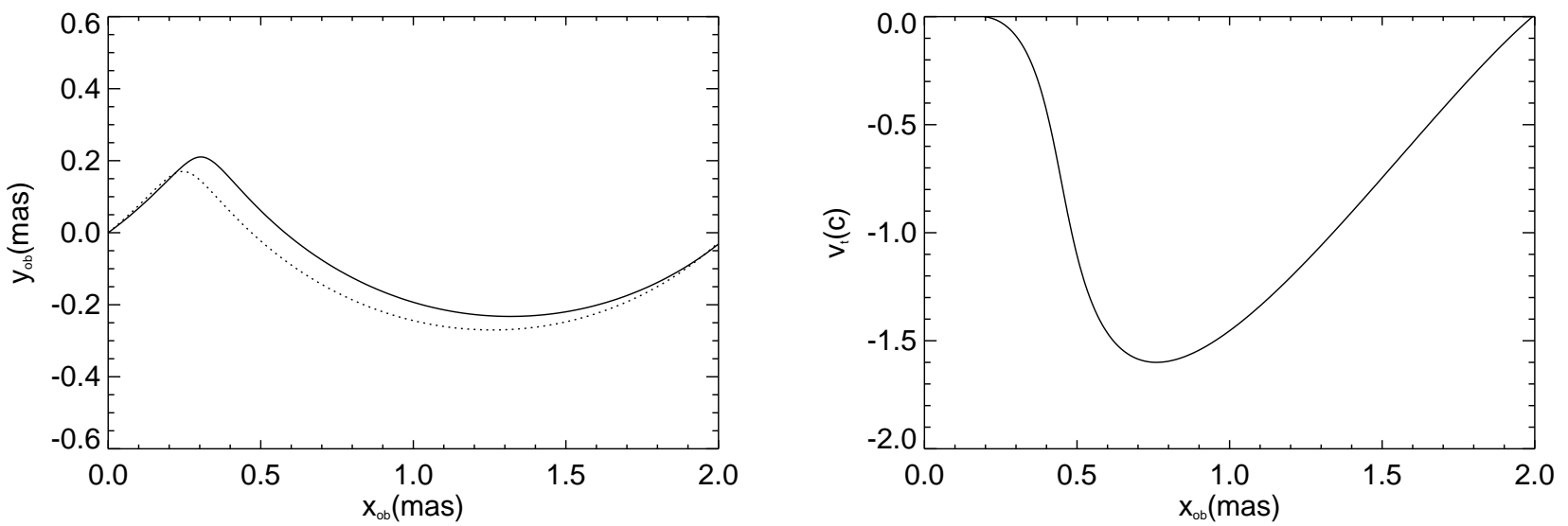

FIG. 18.- Ridge-line motion with at $3^{\circ}$ viewing angle alignment. the ridge-line from the first epoch is the solid line and second epoch is the dotted line. The time interval between them is 2.11 years. On the right, the transversal velocity versus the observed (projected) axial position, obtained from the transversal displacement measured at a fixed distance to the core.

In this work we have presented evidence for the observed helical structures in the jet of $0836+710$ being real, and not artifacts produced by the observational systematic errors or to insufficient uv-coverage. The evidence comes from the same wavelengths being found at all epochs, frequencies and observing arrays. We have shown that shorter wavelengths develop on top of the longer ones and, if their positions along the jet are considered to follow a wave structure, the resulting location has to be the result of the superposition of the different waves and will also depend on the dominant wave at a given position and time.

The fact that shorter wavelengths develop on top of longer wavelengths provides a natural way to explain the misalignment between parsec and kiloparsec scale helical jets seen at small angles to the line of sight and we conclude that at the highest frequencies we may be seeing a small region of the jet flow concentrated around the pressure maxima that we associated with the intensity maximum ridge-line. This identification is bolstered by $15 \mathrm{GHz}$ VLBA observations, in which we are able to resolve the jet, that show the position of the ridge-line. This identification is bolstered by $15 \mathrm{GHz}$ VLBA obser- vations, in which we are able to resolve the jet, that show the position of the ridge-line as not coincident with the center of jet. The opening angle of the jet is found to be the same at all frequencies, with no apparent frequency stratification.

In the light of our results, we reinterpret the results of Perucho \& Lobanov (2007), correcting a mistake in the calculations and in the possible identification of the triggered instability modes. We confirm the general conclusions obtained in that work to the extent that our present study allows but we show that alternative explanations are still possible. Future work will include a detailed discussion on this topic.

We have pointed out that measuring the velocities of the waves is crucial to connecting observed wavelengths to the intrinsic wavelengths responsible for the observed structures. We have tried to measure the wave velocities but the errors associated with the resolution at the different frequencies makes this impractical.

We have also presented a method for modeling helical patterns propagating at relativistic speeds in extragalactic jets. Future work should include the application of this method to the observed structures. Finally, we have 
proposed a possible explanation for moderate transversal superluminal motions with important implications regarding the nature of the jet-core. In particular, we have shown that the radio core at any frequency could be undergoing transversal motion, implying its possible association with wave motion. Moreover, the wave-pattern obtained for the transversal velocity in the $0836+710$ jet indicates that moving waves are present in the jet.

Large angular resolution achieved by future spaceVLBI projects, combined with high precision VLBI astrometry, may help to improve the resolution and make it possible to accurately determine the velocities of the structures in helical jets in nearby AGN.

The authors thank the referee for his/her constructive criticism that has helped to improve the manuscript. This research has made use of data from the MOJAVE database that is maintained by the MOJAVE team Lister et al. (2009). MP acknowledges financial support by the Spanish "Minister de Ciencia e Innovación"
(MICINN) grants AYA2010-21322-C03-01, AYA201021097-C03-01 and CONSOLIDER2007-00050, and by the "Generalitat Valenciana" grant "PROMETEO-2009103". MP acknowledges support from the postdoctoral fellowship "Beca Postdoctoral d'Excel-lència" of "Generalitat Valenciana", a postdoctoral fellowship in MaxPlanck-Insitut for Radioastronomy in Bonn and MICINN through a "Juan de la Cierva" contract. This paper was begun when YYK was a Research Fellow of the Alexander von Humboldt Foundation. YYK is partly supported by the Russian Foundation for Basic Research (grant 1102-00368). YYK thanks hospitality from the staff in the University of Valencia and grant CONSOLIDER200700050. PEH acknowledges support from NSF award AST-0908010 and NASA award NNX08AG83G to the University of Alabama. IA acknowledges funding support from MICINN grant AYA-2010-14844, CEIC grant P09-FQM-4784, NASA award NNX08AV65G, and NSF award AST-0907893.

Facilities: EVN, VLBA, VSOP

\section{REFERENCES}

Agudo, I., Bach, U., Krichbaum, T.P., et al. 2007, A\&A, 476, L17 Agudo, I., Jorstad, S.G., Marscher, A.P., Larionov, V.M., Gómez, J.L., Wiesemeyer, W., Thum, C., Gurwell, M., Heidt, J., D'Arcangelo, F.D. 2010, at Fermi meets Jansky AGN at Radio and Gamma-Rays proceedings. editors: Savolainen, T., Ros, E. Porcas, R.W. \& Zensus, J.A. June 21-23, 2010, Bonn, Germany Agudo, I., Jorstad, S.G., Marscher, A.P., et al. 2011, ApJ, 726, L13

Bach, U., Villata, M., Raiteri, C.M., et al. 2006, A\&A, 456, 105

Caproni, A., Monteiro, H., Abraham, Z. 2009, MNRAS, 399, 1415

Condon, J. J. 1997, PASP, 109, 166

Gong, B. 2007, MNRAS, 389, 315

Hardee, P.E., Cooper, M.A., Clarke, D.A. 1994, ApJ, 424, 126

Hardee, P.E. 2000, ApJ, 533, 176

Hardee, P.E. 2003, ApJ, 597, 798

Hardee, P.E., Walker, R.C., Gómez, J.L. 2005, ApJ, 620, 646

Hardee, P.E. 2006, in "Relativistic Jets: The Common Physics of AGN, Microquasars and Gamma-Ray Bursts". Eds.: P.A. Hughes and J.N. Bregman. AIP Conference Proceedings, 856, 57

Hardee, P.E., 2011, IAU Symposium 275: Jets at all Scales, eds. G. Romero, R. Sunyaev \& T. Belloni, 275, 41

Hardee, P.E., \& Eilek, J.A. 2011, ApJ, in press

Hummel, C.A., Mruxlow, T.W.B., Krichbaum, T.P., et al. 1992, A\&A, 266, 93

Kovalev, Y.Y., Lobanov, A.P., Pushkarev, A.B., Zensus, J.A. 2008, A\&A, 483, 759

Krichbaum, T.P., Hummel, C.A., Quirrenbach, A., et al. 1990, A\&A, 230, 271

Lister, M.L., Kellermann, K.I., Vermeulen, R.C., Cohen, M.H., Zensus, J.A., Ros, E. 2003, ApJ, 584, 135

Lister, M., Aller, H.D., Aller, M.F., et al. 2009, AJ, 137, 3718

Lobanov, A.P. 1998, A\&A, 330, 79

Lobanov, A.P., and Zensus, J.A. 2001, Science, 294, 128

Lobanov, A.P., and Roland, J. 2005, A\&A, 431, 831

Lobanov, A.P., Krichbaum, T.P., Witzel, A., et al. 1998, A\&A, 340,60
Lobanov, A.P., Krichbaum, T.P., Witzel, A., Zensus, J.A. 2006, PASJ, 58, 253

Marscher, A.P., Jorstad, S.G., D'Arcangelo, F.D. et al. 2008, Nature, 452, 966

Martí-Vidal, I., Marcaide, J.M., Alberdi, A., Pérez-Torres, M.A.,

Ros, E., Guirado, J.C. 2011, A\&A, in press (arXiv:1107.0704v2)

McKinney, J.C., and Blandford, R.D. 2009, MNRAS, 394L, 126

Mignone, A., Rossi, P., Bodo, G., Ferrari, A., Massaglia, S. 2010, MNRAS, 402,7

Mizuno, Y., Hardee, P.E., Nishikawa, K.-I. 2007, ApJ, 662, 835

Mizuno, Y., Lyubarsky, Y., Nishikawa, K.-I., Hardee, P.E. 2009 ApJ, 700, 684

Mizuno, Y., Hardee, P.E., Nishikawa, K.-I. 2011, ApJ, 734, 19

Otterbein, K., Krichbaum, T.P., Kraus, A., et al. 1998, A\&A, 334, 489

Perucho, M., 2011, in the Proceedings of High Energy

Phenomena in Relativistic Outflows III (HEPRO-III), eds. J.M. Paredes, M. Ribó, F.A. Aharonian and G.E. Romero, IJMPD, accepted (arXiv:1109.2403).

Perucho, M., Martí, J. M., Hanasz, M. 2005,A\&A, 443, 863

Perucho, M., Lobanov, A.P., Martí, J.M., Hardee, P.E. 2006, A\&A, 456, 493

Perucho, M., Hanasz, M., Martí, J. M., Miralles, J.A. 2007, Phys. Rev. E, 75, 056312

Perucho, M., Martí, J. M., Cela, J. M., Hanasz, M., de la Cruz, R., Rubio, F. 2010, A\&A, 519, A41

Perucho, M., Lobanov, A.P. 2007, A\&A, 469, L23

Perucho, M., Lobanov, A.P. 2011, A\&AErratum, 553C, 2

Pushkarev, A.B., Kovalev, Y.Y., 2011, A\&A, submitted

Pushkarev, A.B., Kovalev, Y.Y., Lister, M.L., Savolainen, T. 2009, A\&A, 507, L33

Savolainen, T., Wiik, K., Valtaoja, E., Tornikoski, M. 2006, A\&A, 446, 71

Sokolovsky, K.V., Kovalev, Y.Y., Pushkarev, A.B., Lobanov, A.P. 2011, A\&A, 532, A38

Stirling, A.M., Cawthorne, T.V., Stevens, J.A., et al. 2003,

MNRAS, 341, 405

Wright, N. 2006, PASP, 118, 1711 